\title{
Proficient Photocatalytic and Sonocatalytic Degradation of Organic Pollutants Using CuO Nanoparticles
}

\author{
Moondeep Chauhan, ${ }^{1}$ Navneet Kaur, ${ }^{1}$ Pratibha Bansal, ${ }^{1}$ Rajeev Kumar, ${ }^{1}$ \\ Sesha Srinivasan ${ }^{(D)}{ }^{2}$ and Ganga Ram Chaudhary ${ }^{1}{ }^{1}$ \\ ${ }^{1}$ Department of Chemistry \& Center of Advanced Studies in Chemistry and Department of Environmental Studies, Panjab University, \\ 160014, Chandigarh, India \\ ${ }^{2}$ Department of Natural Sciences, Division of Science, Arts and Mathematics (SAM), Florida Polytechnic University, \\ Florida-33805, USA
}

Correspondence should be addressed to Sesha Srinivasan; ssrinivasan@floridapoly.edu

Received 26 September 2019; Revised 19 March 2020; Accepted 4 April 2020; Published 10 June 2020

Academic Editor: Silvia Licoccia

Copyright (c) 2020 Moondeep Chauhan et al. This is an open access article distributed under the Creative Commons Attribution License, which permits unrestricted use, distribution, and reproduction in any medium, provided the original work is properly cited.

\begin{abstract}
In recent years, due to the advancement in nanotechnology, advanced oxidation processes (AOPs), especially sonocatalysis and photocatalysis, have become a topic of interest for the elimination of pollutants from contaminated water. In the research work reported here, an attempt has been made to study and establish a physicochemical mechanism for the catalytic activity of copper oxide nanoparticles ( $\mathrm{CuO} \mathrm{NPs}$ ) in AOPs using the degradation of dyes as model contaminants. CuO NPs exhibited brilliant sonocatalytic and photocatalytic activities for the degradation of a cationic dye (Victoria Blue) as well as an anionic dye (Direct Red 81). The degradation efficiency of CuO NPs was calculated by analysing the variation in the absorbance of dye under a UV-Vis spectrophotometer. The influence of different operating parameters on the catalytic activity of CuO NPs, such as the amount of catalysts dose, $\mathrm{pH}$ of the solution, and the initial dye concentration, was thoroughly investigated. In addition, the kinetic process for the degradation was also examined. It was observed that both dyes exhibited and followed the pseudofirst-order kinetics relation. The rate constant for sonocatalysis was high as compared to photocatalysis. The rate constant for both sonocatalysis and photocatalysis was successfully established, and reusability tests were done to ensure the stability of the used catalysts. To get an insight into the degradation mechanism, experiments were performed by using $\mathrm{OH}$ radical scavengers. The efficacy of $\mathrm{CuO}$ NPs for dye decolorization was found to be superior for the sonocatalyst than the photocatalyst.
\end{abstract}

\section{Introduction}

Although awareness of the need to avoid contamination of the environment has increased, large amounts of synthetic or natural pollutants, such as dyes, heavy metals, pharmaceutical waste, and agrotoxic waste, like pesticides, continue to be discharged in the environment by different industries [1]. Among these, synthetic organic dyes are one of the leading contributors to environmental pollution since dyes are used by various industries, such as plastic, paper, textile, leather, cosmetic, and food industries [2,3]. As dyes have a complex aromatic structure and are difficult to degrade, their presence in the aquatic environment can cause problems, such as color change, odor alteration, eutrophication, underoxygenation, and bioaccumulation [4]. Therefore, their removal from industrial wastewater is highly desirable to achieve concentrations below environmentally accepted levels for safe disposal.

At present, several techniques have been developed for the removal of pollutants from wastewater, such as adsorption, chemical coagulation, filtration, sedimentation, and advance oxidation process (AOPs) [5-8]. Recently, AOPs are gaining significant importance due to their ability to generate a sufficient number of highly reactive radicals for effective water decontamination. Among numerous AOPs, photocatalysis and sonocatalysis with metal oxide nanoparticles (NPs) have 
attracted more attention as efficient techniques for the degradation of toxic organic contaminants [9-18].

In the case of photocatalytic degradation, irradiation with photon energy greater than the band gap of NPs leads to the formation of electron and hole pairs, which further helps in the generation of highly reactive oxygen species (ROS) that eventually participate in the degradation of toxic compounds [19-23]. It is evident that the limiting factor for catalytic degradation depends upon the ability to form greater numbers of ROS [24]. On the other hand, sonocatalytic degradation of toxic compounds is caused by a chemical effect of ultrasonic (US) waves which arises from acoustic cavitation, i.e., formation, growth, and collapse of bubbles in a liquid [25]. The transient bubble collapse generates a localized hot spot with a very high temperature (near about $5000 \mathrm{~K})$ and pressure ( 500 bars). Under such extreme conditions, thermal decomposition of water takes place, resulting in the formation of highly reactive radical species such as $\cdot \mathrm{OH}, \cdot \mathrm{H}$, and $\cdot \mathrm{O}$, which can oxidize and degrade organic contaminants in water [26].

In keeping with environmental concerns, there is a significant shift towards the use of nanoparticles in developing green and environmentally friendly approaches in synthetic chemistry [27]. In our previous work, we have reported the synthesis of $\mathrm{CuO}$ NPs and further investigated their catalytic activity in the synthesis of xanthenes [28]. The synthesis of $\mathrm{CuO}$ NPs was carried out with microwaves, using a greener approach, which has achieved many advantages in the synthesis of xanthenes, such as low energy, cost effectiveness, shorter processing time, less pollution, utilization of a green solvent, and a lower reaction temperature with high yield as compared to other methods [28]. As determined through X-ray diffraction studies, the as-synthesized NPs were composed of a pure $\mathrm{CuO}$ phase with a monoclinic structure without any peaks of impurity (Figure S1, supplementary data). Particle size, as calculated from FWHM of reflection $(111 / 200)$ of monoclinic $\mathrm{CuO}$ structure using the Debye Scherrer formula, was found to be $18 \mathrm{~nm}$. For determining the morphology, the TEM micrograph of CuO NPs was taken and NPs were observed to be almost spherical in shape, well dispersed, and within the narrow range of size distribution (17-22 nm) (Figure S2, S3, supplementary data) [28].

In the present work, we have explored $\mathrm{CuO}$ NPs as a catalyst for degradation of Victoria Blue (VB) and Direct Red 81 (DR) dyes as model organic contaminants. To find out which energy source is better in terms of the synergistic effect with $\mathrm{CuO}$ NPs for the degradation of dyes, we have used UVvisible light and US waves as sources of energy. The effect of $\mathrm{pH}$, catalyst dosage, and initial dye concentration with respect to contact time has been particularly evaluated. An attempt has also been made to recover the $\mathrm{CuO}$ NPs from the reaction mixture in both cases and to reuse them in subsequent processes. The kinetics rate constant of dyes under both energy sources has been evaluated and found to have significant efficiency.

\section{Experimental Details}

2.1. Materials and Techniques. Victoria Blue (VB) and Direct Red 81 (DR) dyes were obtained from Sigma-Aldrich.
Dimethyl sulfoxide and sodium bicarbonate were purchased from Qualigens, and absolute ethanol (purity 99.9\%) was purchased from Changshu Yangyuan Chemicals, China. All of the chemicals were used as received, and double distilled water was used for the preparation of different solutions. Characterization of recycled nanoparticles was done by transmission electron microscope (TEM) (TEM; Hitachi-H-7500) operating at $80 \mathrm{kV}$. The crystallinity and $\mathrm{X}$ - ray diffraction pattern of the samples were performed at room temperature utilizing X-ray diffraction (XRD; PANanalytical X'Pert Pro) equipped with $\mathrm{Cu}-\mathrm{k} \alpha$ radiation $(\lambda=1.54178 \AA$ ) with A scanning speed of $8 \mathrm{~min}^{-1}$, in the $2 \theta$ range of $0-90^{\circ}$. The zeta potential of the $\mathrm{CuO}$ NPs was examined at different $\mathrm{pH}$ ranges with a Malvern Zetasizer (ZS 90) at room temperature.

2.2. Synthesis and Characterization of $\mathrm{CuO}$ NPs. CuO NPs have been synthesized via a cost-effective and greener approach by using microwave and DI water as the medium. Within 4 minutes of reaction time, very high yields of $\mathrm{CuO}$ NPs were achieved. Detailed procedure and characterization have been reported earlier [5]. To study the photocatalytic and sonocatalytic efficiency of the NPs, surface area of the nanoparticles should be known. BET (Branauer-EmmettTeller) surface area of CuO NPs was studied using Quantachrome Instrument (version 3.01).

2.3. Sonocatalytic and Photocatalytic Degradation Reaction Procedures. To evaluate the catalytic (photocatalytic and sonocatalytic) activity of $\mathrm{CuO}$ NPs and to further understand the degradation mechanism of both the dyes, batch experiments were performed at room temperature. Before exposing to ultrasonic waves or UV-visible light, the suspensions (dye+NPs) were kept in dark conditions and stirred for 10 minutes to get the adsorption-desorption equilibrium of the dye on the $\mathrm{CuO}$ NPs. Therefore, the effect of adsorption during sonocatalysis or photocatalysis could be calculated accurately.

In case of sonocatalysis, these suspensions were placed in an ultrasonic bath apparatus (PCI Analytics) for a definite interval of time. After ultrasonic irradiation, $\mathrm{CuO}$ NPs were separated from the suspension by centrifugation and percent degradation was calculated by measuring the change in the absorbance of dye using UV-Vis spectrophotometer. In the case of photocatalysis, the experiment was carried out by placing the suspended solution in a UV-visible photoreactor $\left(125 \mathrm{~W}, 198.4 \mathrm{mWS}^{-2}\right)$ for a definite time interval. After exposure to UV-visible radiations for a desired time interval, samples were withdrawn from the chamber and $\mathrm{CuO}$ NPs were separated from the suspension by centrifugation. The degradation rates of the dyes at given time intervals were calculated by measuring the changes in the absorption profile at their maximum absorbance wavelength $\left(\lambda_{\max }\right)$ using a UVvis spectrophotometer (UV 2100, Shimadzu). The degradation observations were performed under ambient conditions and the temperature was kept at $25-30^{\circ} \mathrm{C}$. Typically, $20 \mathrm{ml}$ of $10 \times 10^{-5} \mathrm{M}$ solutions of both dyes with initial solution $\mathrm{pH} 10$ for VR dye and 4.0 for DR dye were taken, and $25 \mathrm{mg}$ of catalyst dose was taken in general throughout the whole investigation. For sonocatalysis, ultrasonic irradiation of $50 \mathrm{kHz}$ frequency and $50 \mathrm{~W}$ power were used, and for photocatalysis, 


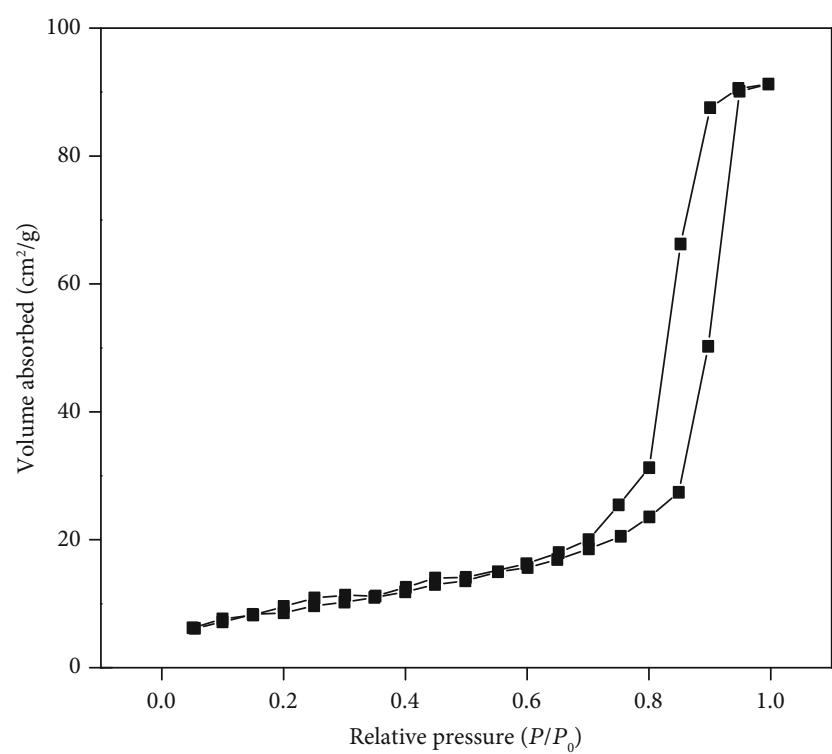

(a)

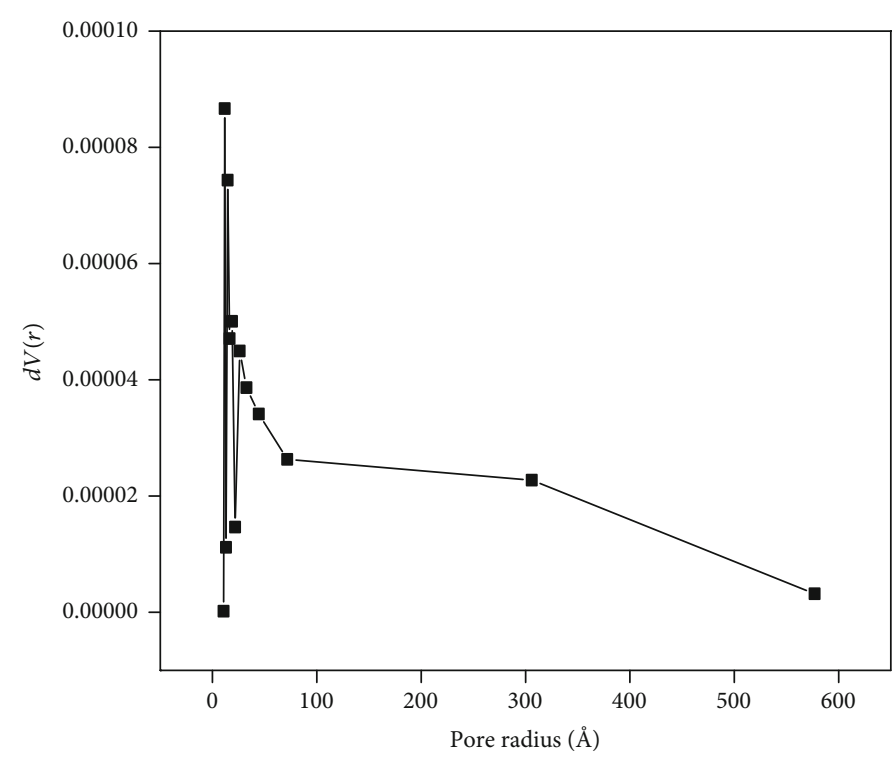

(b)

FIGURE 1: (a) $\mathrm{N}_{2}$ adsorption-desorption BET isotherms and (b) BJH pore size distributions (from the adsorption branch of the isotherms) of synthesized $\mathrm{CuO}$ NPs.

at UV-visible irradiation frequency $198.4 \mathrm{mWS}^{-2}$ and $125 \mathrm{~W}$ power. In addition, the $\mathrm{pH}$ of the dye solutions was adjusted to required $\mathrm{pH}$ by adding $0.1 \mathrm{M} \mathrm{HCl}$ or $\mathrm{NaOH}$ solution. The $\mathrm{pH}$ value of solutions was measured by a CyberScan 510 digital $\mathrm{pH}$ meter.

The following equation (equation (1)) was used for calculating the degradation efficiency (percent degradation) of dyes:

$$
\text { Degradation efficiency }(\%)=\frac{C_{0}-C_{t}}{C_{0}} \times 100
$$

where $C_{0}$ is the initial concentration of the dye before irradiation and $C_{t}$ is the concentration of the dye at a certain reaction time $t$ ( $\mathrm{min})$ after irradiation. The sonocatalytic activity of $\mathrm{CuO}$ NPs, in terms of the degradation of VB and DR, was compared with that of the photocatalytic activity of CuO NPs under UV-visible irradiation. Multiple factors are responsible for the sonocatalytic efficiency of $\mathrm{CuO}$ NPs which are difficult to quantify individually. Moreover, sonocatalysis and photocatalysis are two completely different phenomenon; therefore it is hard to find a correlation factor between the US and UV-Vis energy sources.

\section{Results and Discussion}

3.1. Characterization of $\mathrm{CuO} N$ Ps. The BET isotherms and their relative Barret-Joyner-Halender $(\mathrm{BJH})$ pore size distributions obtained from the adsorption branch of the isotherms of the CuO NPs are displayed in Figure 1(a). It was observed that the prepared $\mathrm{CuO}$ NPs possess a mesoporous surface as the isotherms are of type IV. The multipoint BET-specific surface area for $\mathrm{CuO}$ NPs was found to be $49.33 \mathrm{~m}^{2} \mathrm{~g}^{-1}$. The $\mathrm{CuO}$ NPs were found to have an average pore radius of $1.1764 \mathrm{~nm}$ (Figure 1(b)).
3.2. Percentage Degradation of $V B$ and $D R$ under Different Processes. The degradation efficiency of VB ( $\mathrm{pH} 10)$ and DR ( $\mathrm{pH} 4)$ was investigated by exposing the dye solution to $\mathrm{UV}$-visible irradiation or ultrasonication in the absence and in the presence of CuO NPs (25 mg). Both dyes undergo negligible decomposition under direct UV-visible irradiation or US radiation in the absence of $\mathrm{CuO}$ NPs. On the other hand, the presence of $\mathrm{CuO}$ NPs showed excellent results as a photocatalyst and sonocatalyst in the degradation of both dyes (Figure 2).

3.3. Effect of $p H$. The $\mathrm{pH}$ of the solution is an important parameter that plays a significant role in the sonocatalytic and photocatalytic degradation of various target pollutants $[13,29]$. The zeta potential of the $\mathrm{CuO} N \mathrm{NP}$ dispersions was measured over $\mathrm{pH}$ values ranging from 2 to 10 , and the corresponding $\mathrm{pHzpc}$ was found to be $\sim 8.5$ (Figure S4 supplementary data). The effect of $\mathrm{pH}$ on the photocatalytic activity of $\mathrm{CuO}$ was tested using acidic, neutral, and basic media, and the results are shown in Figures 3 and 4, respectively. It was observed that $\mathrm{CuO} N \mathrm{NP}$ in both degradation processes follow a pseudo-first-order kinetics at different $\mathrm{pH}$ [30]. The equation used is as follows:

$$
\ln \frac{C_{0}}{C_{t}}=k t
$$

where $k$ is the pseudo-first-order rate constant $\left(\mathrm{min}^{-1}\right), C_{0}$ is the initial concentration (at time $t=0$ ) of the dye, and $C_{t}$ is the concentration of the dye at reaction time $t$. As can be seen in Table 1 , rate constant $k$ value increases for the $\mathrm{VB}$ dye from $\mathrm{pH} 4$ to $\mathrm{pH} 10$, and for the $\mathrm{DR}$ dye, the $k$ value decreases from $\mathrm{pH} 10$ to $\mathrm{pH} 4$. Thus, the increase in $\mathrm{pH}$ leads to an enhanced degradation rate of $\mathrm{VB}$ dye and it reached maximum at $\mathrm{pH} 10$. However, in the case of the DR dye, 


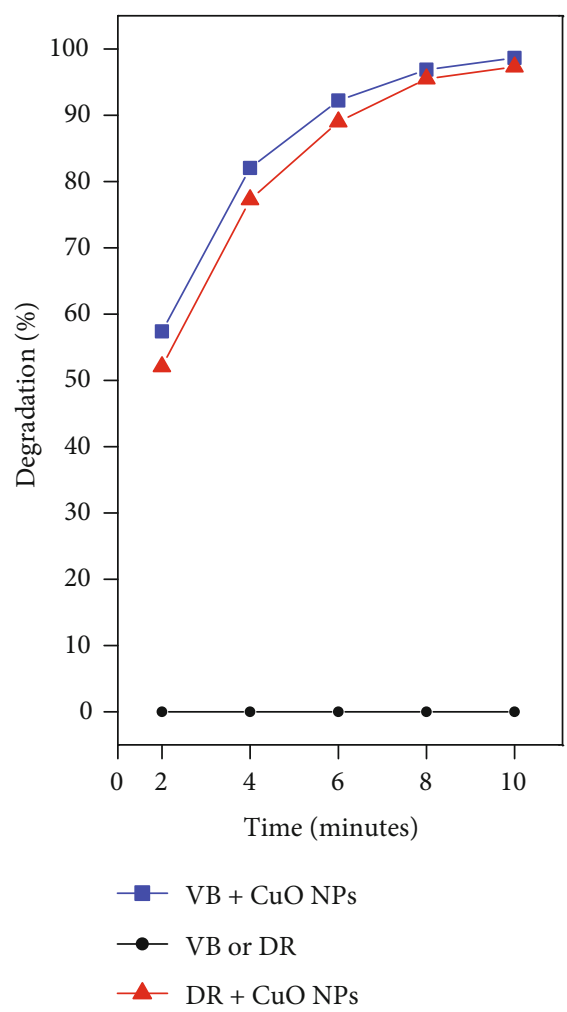

(a)

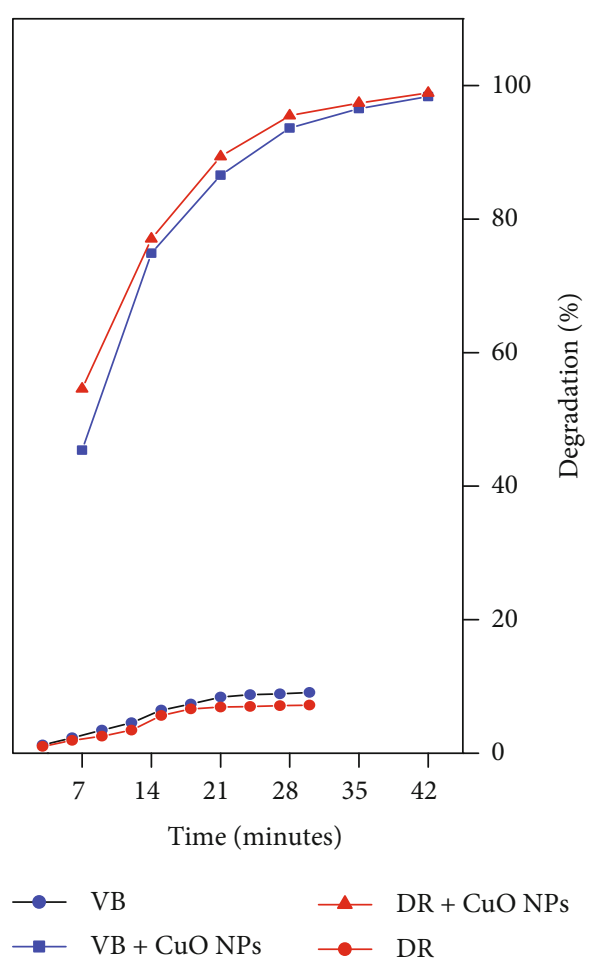

(b)

Figure 2: Degradation of VB and DR dye treated with different process $10 \times 10^{-5} \mathrm{M}$ dye solution, CuO NP is $25 \mathrm{mg}$. (a) US irradiation. (b) UV-visible irradiation.

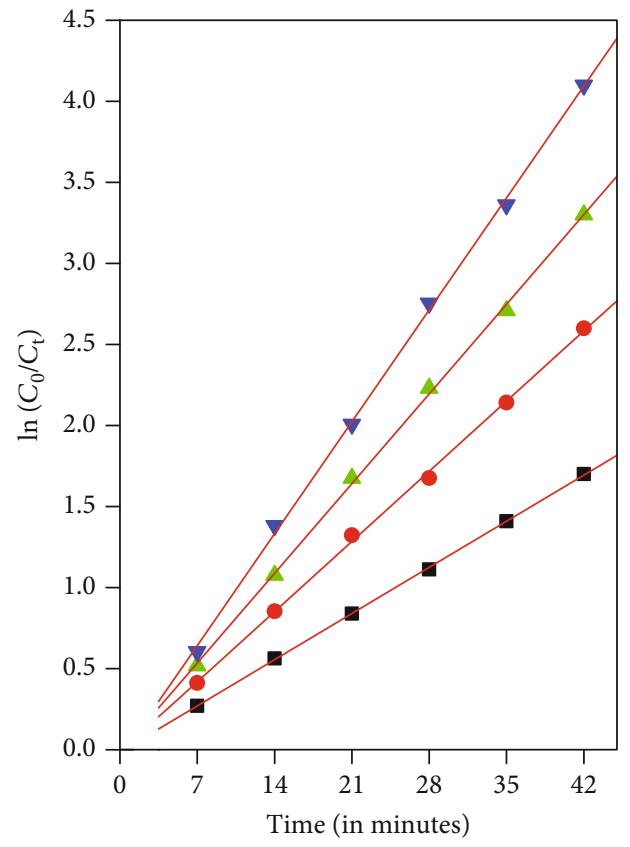

- $4 \mathrm{pH}$

$6 \mathrm{pH}$ $\triangle 8 \mathrm{pH}$

$\nabla 10 \mathrm{pH}$

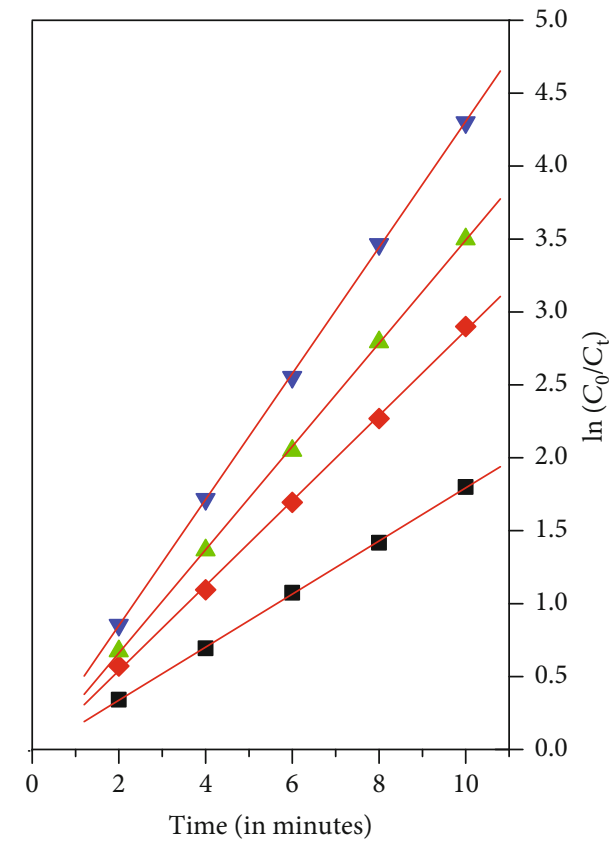

- $4 \mathrm{pH}$

$\triangle 8 \mathrm{pH}$

$\nabla 10 \mathrm{pH}$

(b)

FIgURE 3: Effect of pH on degradation of VB under (a) UV-visible irradiation and (b) US irradiation. 


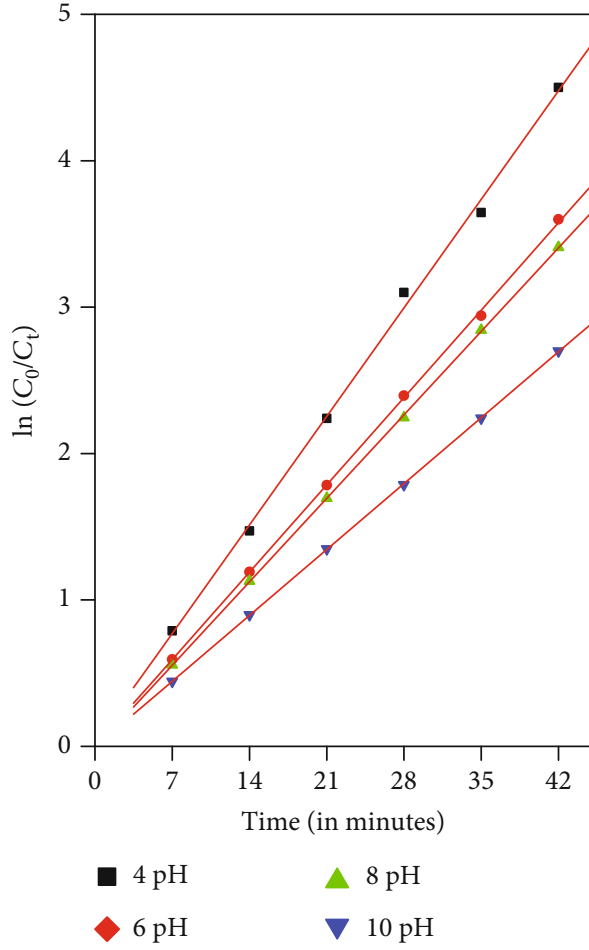

(a)

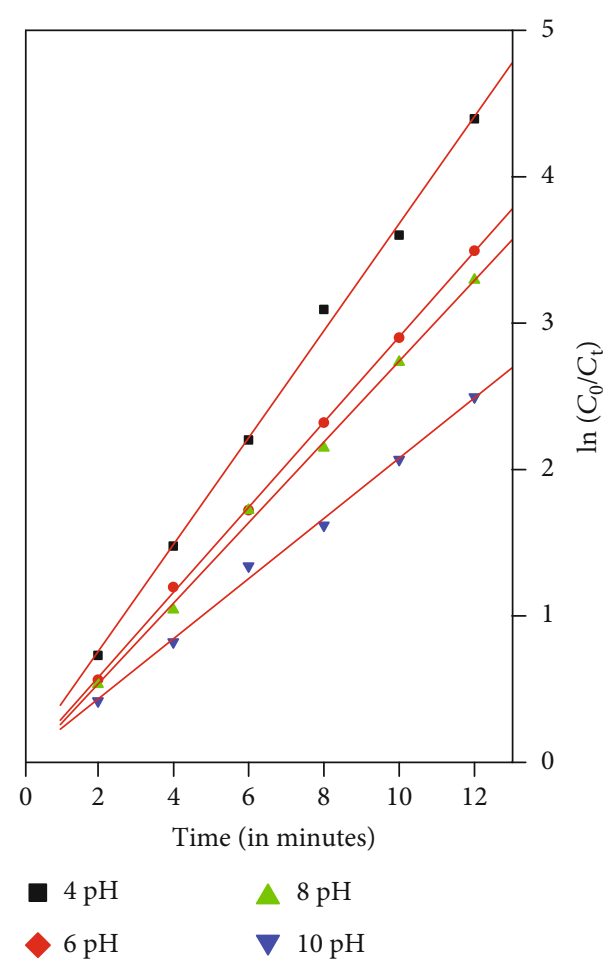

(b)

FIGURE 4: Effect of $\mathrm{pH}$ on degradation of DR under (a) UV-visible irradiation and (b) under US irradiation.

TABLE 1: Pseudo-first-order kinetics rate constants for the degradation of VB and DR under both sources of energy and for different pH.

\begin{tabular}{|c|c|c|c|c|c|}
\hline Dyes & $\mathrm{pH}$ & Sonocatalysis $k\left(\mathrm{~min}^{-1}\right)$ & Photocatalysis $k\left(\mathrm{~min}^{-1}\right)$ & $R$ sonocatalysis & $R$ photocatalysis \\
\hline \multirow{4}{*}{ Victoria Blue (VB) } & 4 & 0.182 & 0.04067 & 0.99986 & 0.99991 \\
\hline & 6 & 0.29153 & 0.06185 & 0.99955 & 0.99944 \\
\hline & 8 & 0.35388 & 0.07903 & 0.9999 & 0.99959 \\
\hline & 10 & 0.43209 & 0.09861 & 0.99992 & 0.99954 \\
\hline \multirow{4}{*}{ Direct Red (DR) } & 4 & 0.3656 & 0.10587 & 0.9985 & 0.99888 \\
\hline & 6 & 0.29098 & 0.08525 & 0.99983 & 0.99982 \\
\hline & 8 & 0.27584 & 0.08144 & 0.99901 & 0.99996 \\
\hline & 10 & 0.20578 & 0.06428 & 0.99825 & 0.99998 \\
\hline
\end{tabular}

the results indicated that the sonodegradation and photodegradation of the dye were maximally efficient in acidic conditions and the optimal $\mathrm{pH}$ was observed at about 4.0.

For VB, higher degradation efficiency in the basic medium must be ascribed to the surface charge properties of $\mathrm{CuO} \mathrm{NPs}$ (zero-point charge $(\mathrm{zpc}) \sim 8.5$ ). The surface charge is positive at $\mathrm{pH}$ values lower than $\mathrm{pH}_{\mathrm{zpc}}$, neutral at $\mathrm{pH}_{\mathrm{zpc}}$ and negative at higher $\mathrm{pH}_{\mathrm{zpc}}$ value. At higher $\mathrm{pH}$, the $\mathrm{CuO} \mathrm{NP}$ surface is negatively charged, so it enhances its interaction with the cationic dye (VB) through electrostatic force of attraction [31] (Scheme 1). Whereas in the case of $\mathrm{DR}$, at lower $\mathrm{pH}$, the surface of $\mathrm{CuO}$ NPs is positively charged so there is an enhancement of electrostatic force of attraction with the anionic dye (DR). Therefore, there is more degradation of DR dye at $\mathrm{pH} 4$ [32] (Scheme 1).

3.4. Effect of Catalyst Dose. The effect of the catalyst dose on the degradation of $\mathrm{VB}$ and $\mathrm{DR}$ was studied by varying the

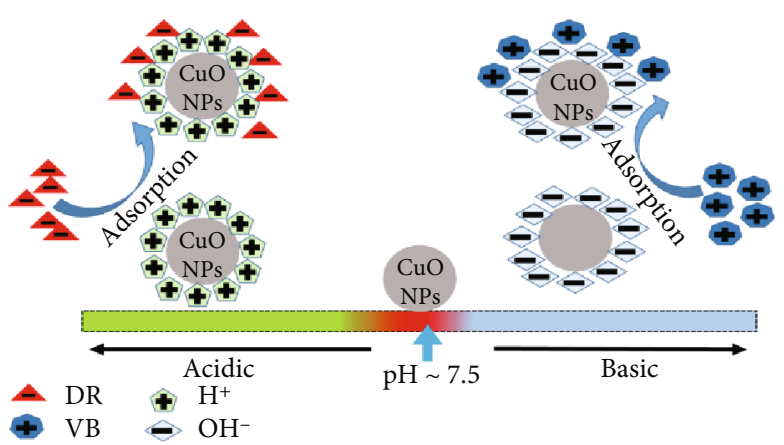

Scheme 1: Role of zero-point charge of NPs on the adsorption of cationic and anionic dyes.

amount of the catalyst from $3 \mathrm{mg}$ to $30 \mathrm{mg}$ in $20 \mathrm{ml}$ of $10 \times 10^{-5} \mathrm{M}$ dye solution at initial $\mathrm{pH}$ of 10 (VB) and 4 (DR) for reaction time, i.e., 7 minutes for sonocatalysis and 


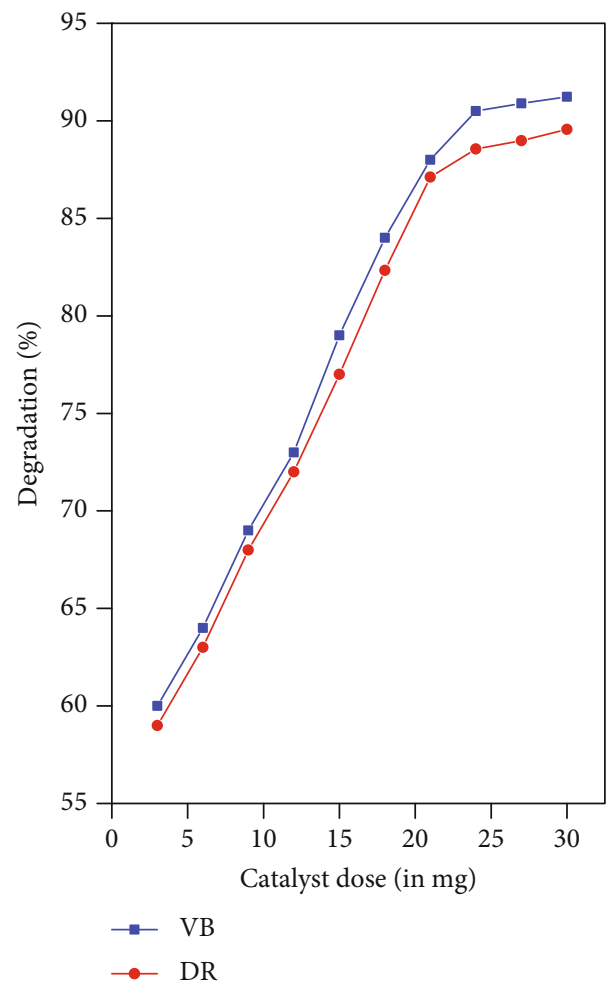

(a)

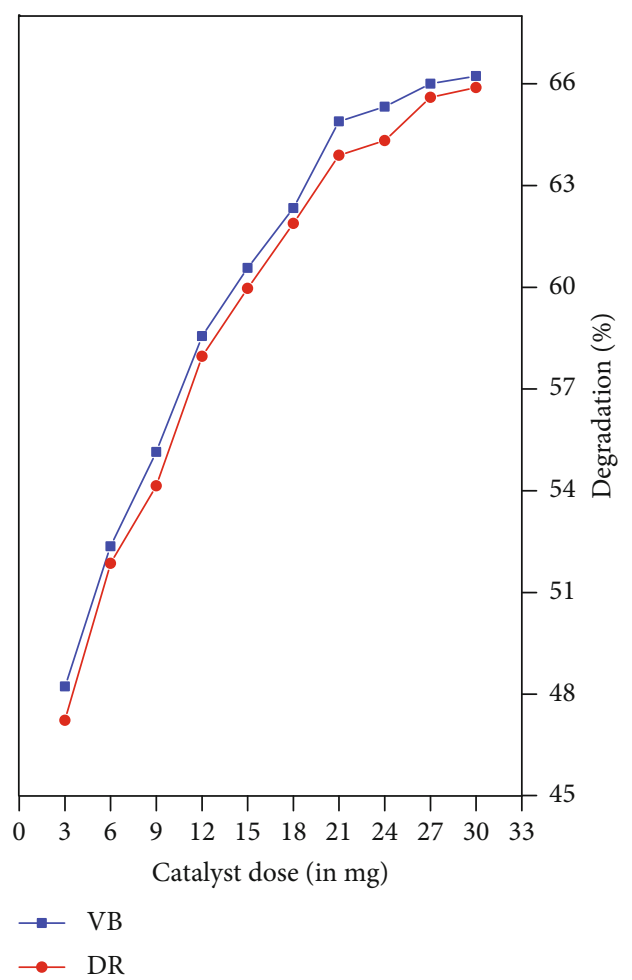

(b)

FIGURE 5: Effect of catalyst dose on degradation of VB and DR for (a) UV-visible irradiation and (b) under US irradiation.

12 minutes for photocatalysis. Figure 5 revealed that increasing the catalyst dose from 3 to $25 \mathrm{mg}$ resulted in an increase in the degradation efficiency, while further increase in the amount of the catalyst dose to $30 \mathrm{mg}$ caused an insignificant increase in the (\%) degradation efficiency (Table S1-S4 supplementary data). Enhanced catalytic activity with increasing $\mathrm{CuO} \mathrm{NP}$ content could be interpreted by two speculations: (1) there will be more availability of active sites and (2) deactivation of activated dye molecules by collision with molecules in the ground state [13]. $\mathrm{OH}$ radicals were the actual oxidants for the decomposition of the VB and DR dyes, whose concentration increases with an increase in catalyst dosage [13].

3.5. Effect of Initial Dye Concentration. From an application point of view, it is important to study the effect of initial VB and DR concentration on its sonocatalytic or photocatalytic degradation efficiency (\%). It was studied by varying initial dye concentration in the range among $10 \times 10^{-5}$, $12 \times 10^{-5}$, and $15 \times 10^{-5} \mathrm{M}$ using an NP dosage of $25 \mathrm{mg}$ in $20 \mathrm{ml}$ of dye solution and at a suitable $\mathrm{pH}$. The results showed that the degradation efficiency (\%) of dyes is inversely proportional to its initial concentration. As a result, increasing the initial dye concentration led to a decrease in the degradation (\%) of dyes (Figures 6 and 7). This negative effect may be caused by the following: (i) The number of dye molecules that was adsorbed on the surface of the catalyst increases with the increasing dye concentration. In addition, at a high dye concentration, a significant amount of UV light or ultrasonic irradiation may be absorbed by the dye molecules rather than by the catalyst, thereby reducing the efficiency of the catalytic reaction. (ii) The generation of ${ }^{\circ} \mathrm{OH}$ radicals on the surface of the catalyst is likely to be reduced since active sites on the surface of the catalyst are occupied by the dye ions. (iii) With the increasing dye concentration, the number of hydroxyl radicals required for the degradation of dye molecules increases, although the formation of hydroxyl radicals remains constant for a given catalyst dosage, irradiation time, and intensity [13].

3.6. Mechanism. In the case of photocatalysis, as UV-visible irradiation falls on semiconducting $\mathrm{CuO}$ NPs, it induces the formation of electron-hole pairs through the photoexcitation of electrons from the valence band to the conduction band (equation (3)) [33].

$$
\mathrm{CuO} \stackrel{\text { UV-visible }}{\longrightarrow} \mathrm{h}^{+}+\mathrm{e}^{-} \text {. }
$$

Photogenerated electrons react with dissolved molecular oxygen, forming a superoxide ion $\mathrm{O}_{2}{ }^{--}$(equation (4)), which further react with a proton to form a hydroperoxide radical $\left(\mathrm{HO}_{2}\right)$ (equation (5)).

$$
\begin{gathered}
\mathrm{e}^{-}+\mathrm{O}_{2} \longrightarrow \mathrm{O}_{2}^{--} \\
\mathrm{O}_{2}^{--}+\mathrm{H}^{+} \longrightarrow \mathrm{HO}_{2}
\end{gathered}
$$




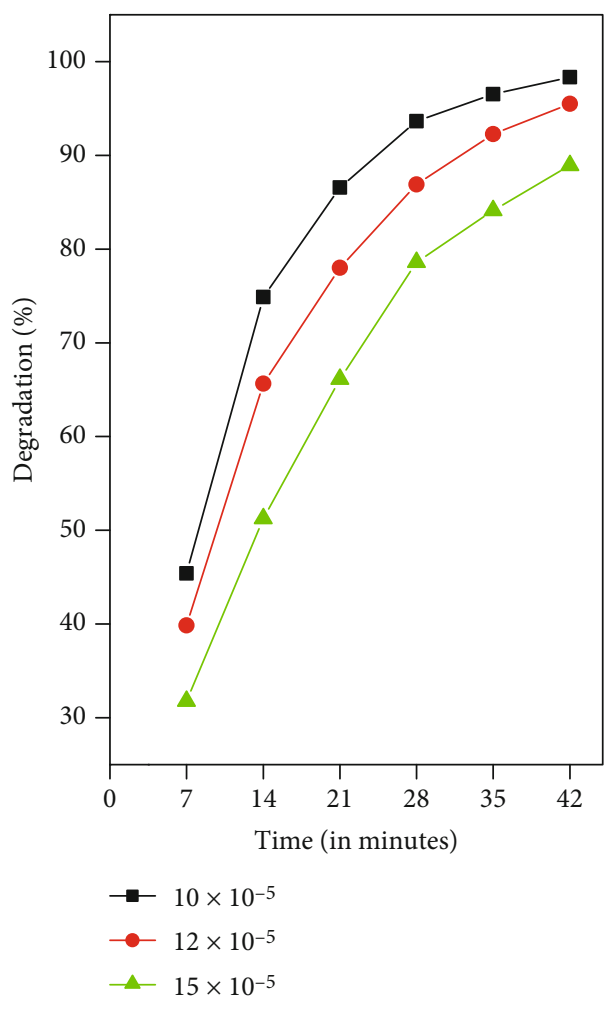

(a)

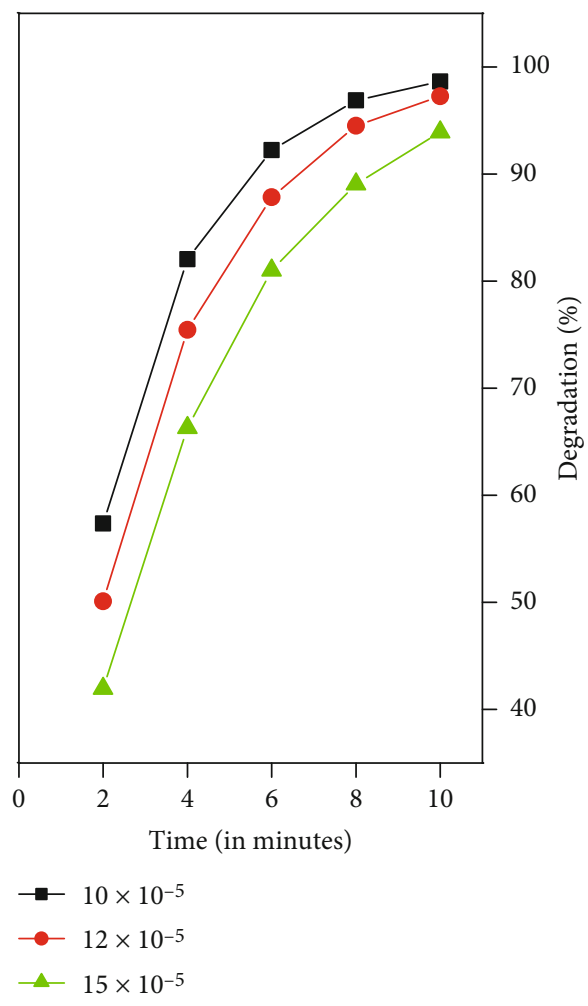

(b)

FIGURE 6: Effect of initial concentration VB dye on degradation under (a) UV-visible irradiation and (b) US irradiation.

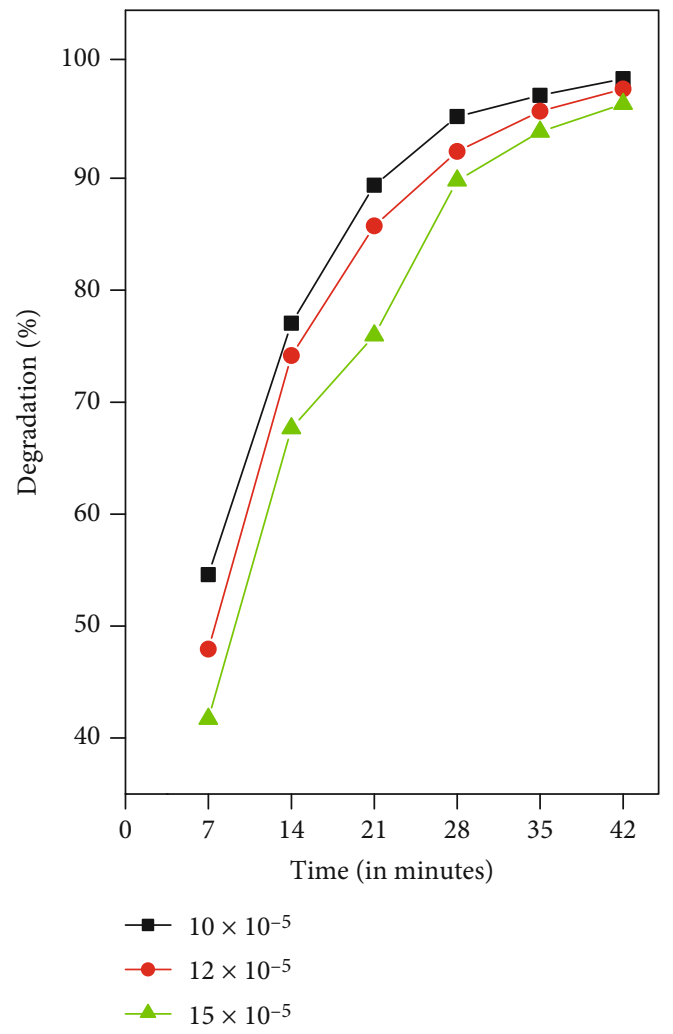

(a)

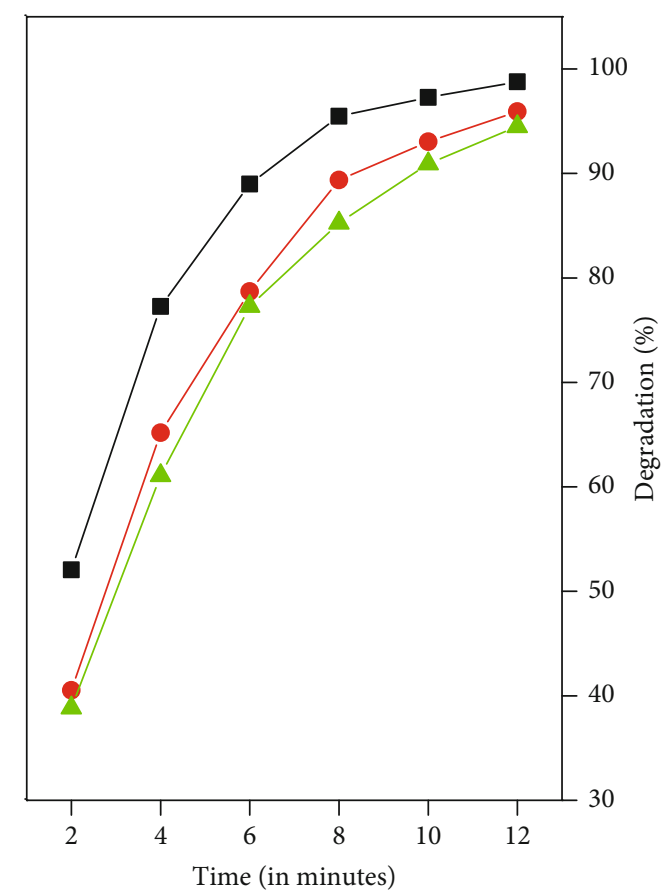

$-10 \times 10^{-5}$

- $12 \times 10^{-5}$

$\simeq 15 \times 10^{-5}$ 


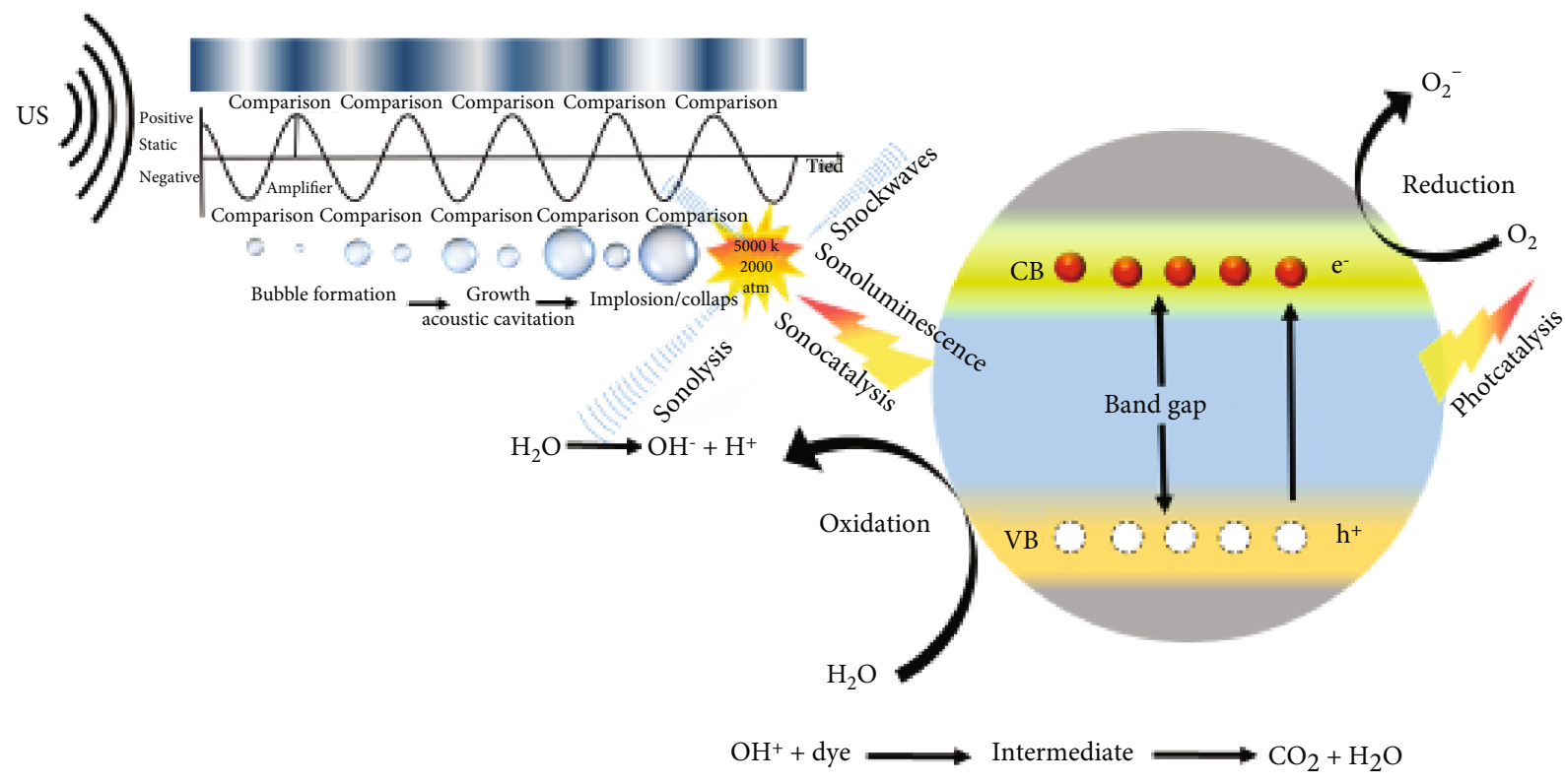

Scheme 2: Schematic diagram representing the photocatalytic and sonocatalytic degradation mechanisms.

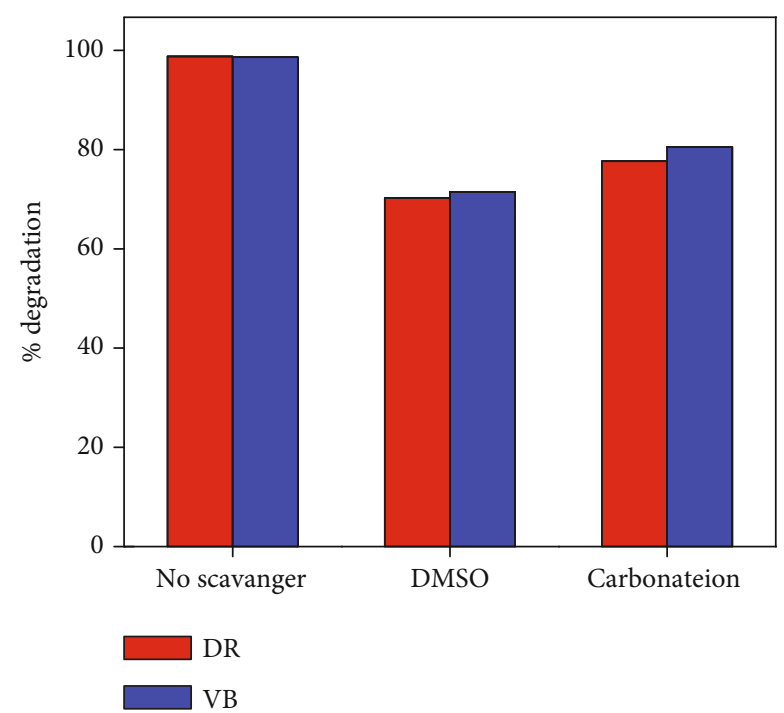

(a)

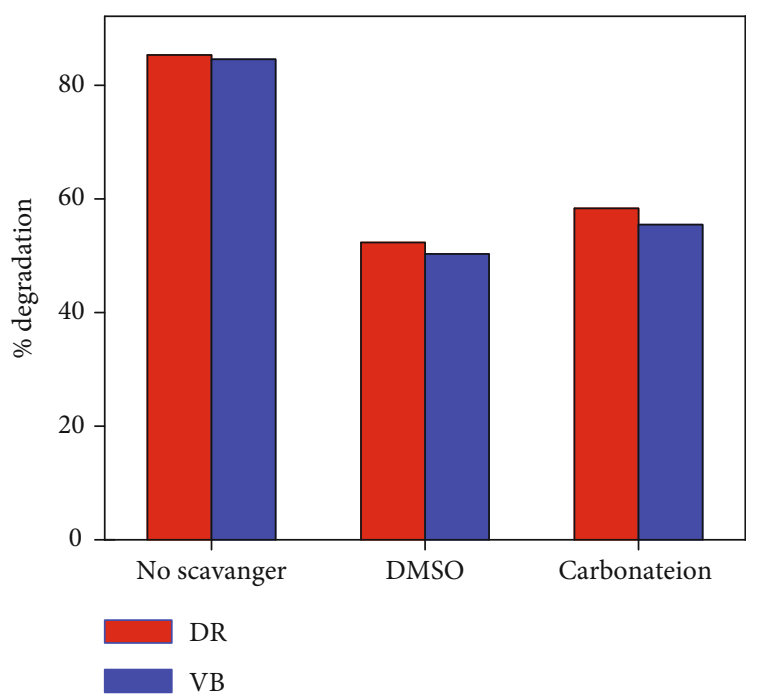

(b)

FIGURE 8: Influence of adding different scavengers (DMSO and carbonate ion). Experimental conditions: scavenger concentration $1 \mathrm{M}$, catalyst weights $30 \mathrm{mg}, \mathrm{pH} 8$ (VB), and 4 (DR) (a) under US irradiation and (b) under UV-visible irradiation.

Simultaneously, photogenerated holes can react with water molecules (equation (6)) and the hydroxide anions $\left(\mathrm{OH}^{-}\right)$to form highly active hydroxyl radicals $(\cdot \mathrm{OH})$ (equation (7)).

$$
\begin{aligned}
& \mathrm{h}^{+}+\mathrm{H}_{2} \mathrm{O} \longrightarrow \mathrm{h}^{+}+\mathrm{HO}^{\circ} \\
& \mathrm{h}^{+}+\mathrm{OH}^{-} \longrightarrow \mathrm{HO}
\end{aligned}
$$

The superoxide and hydroxyl radicals have strong oxidation performance, which can completely oxidize the
$\mathrm{VB} / \mathrm{DR}$ dye molecules into the final products $\mathrm{CO}_{2}$ and $\mathrm{H}_{2} \mathrm{O}$ (equations (8) and (9)).

$\mathrm{VB} / \mathrm{DR}+$ catalyst $-\mathrm{OH} \cdot \stackrel{\text { US/UV-visible }}{\longrightarrow} \mathrm{CO}_{2}+\mathrm{H}_{2} \mathrm{O}+$ inorganic

$\mathrm{VB} / \mathrm{DR}+$ catalyst $-\mathrm{O}_{2}^{--} \stackrel{\text { US/UV-Visible }}{\longrightarrow} \mathrm{CO}_{2}+\mathrm{H}_{2} \mathrm{O}+$ inorganic

On the other hand, the chemical effects of ultrasound are due to the phenomenon of cavitation which is the nucleation, growth, and collapse of bubbles in a liquid 


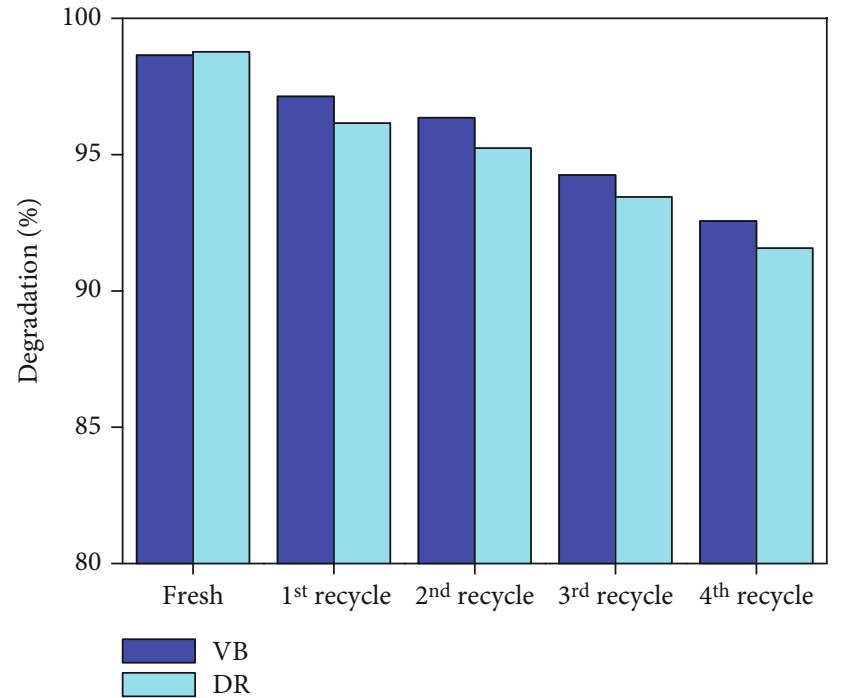

(a)

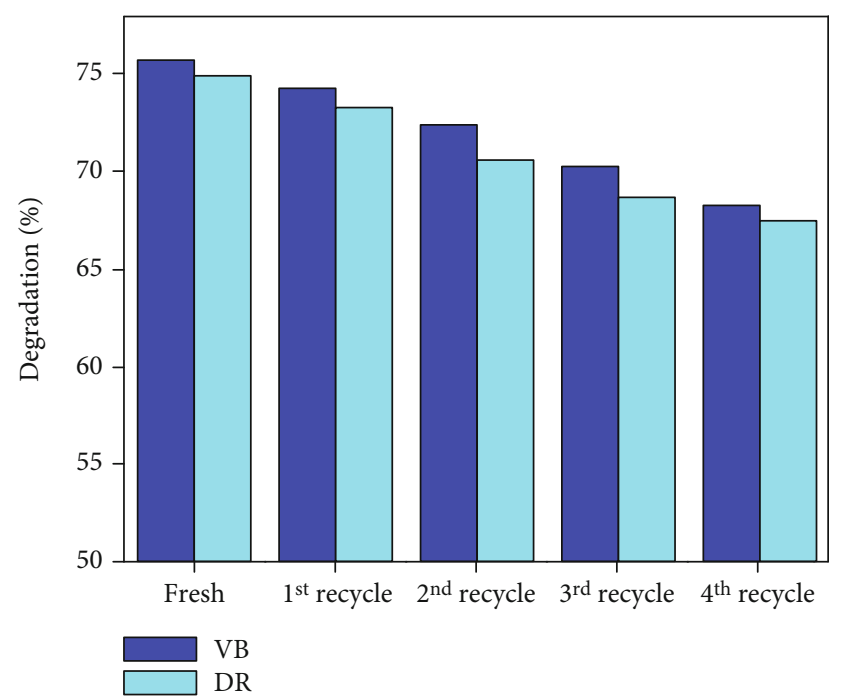

(b)

FIGURE 9: Recyclability of CuO NPs (25 mg) for the degradation of VB ( $\mathrm{pH} 10)$ and $\mathrm{DR}(\mathrm{pH} 4)$ dyes, time 12 minutes under (a) US irradiation and (b) UV-visible irradiations.

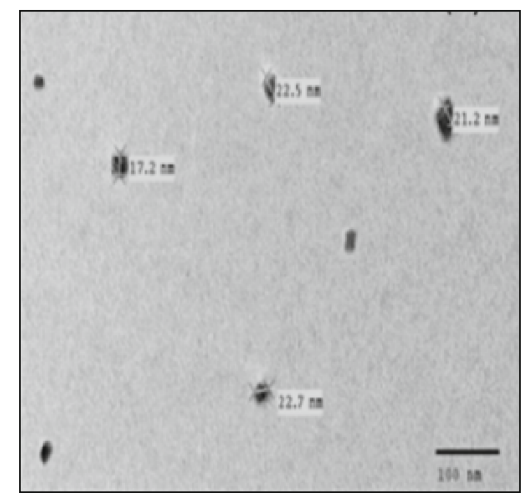

(a)

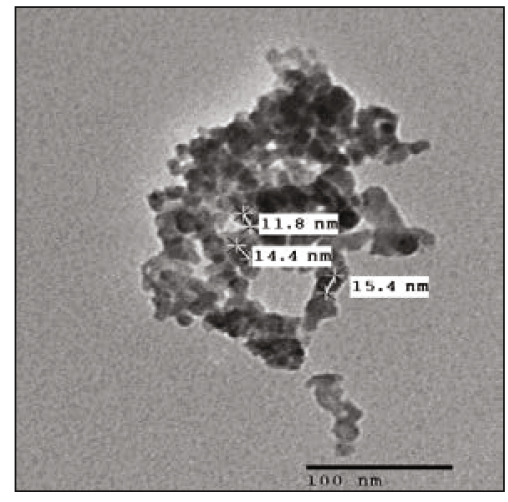

(b)

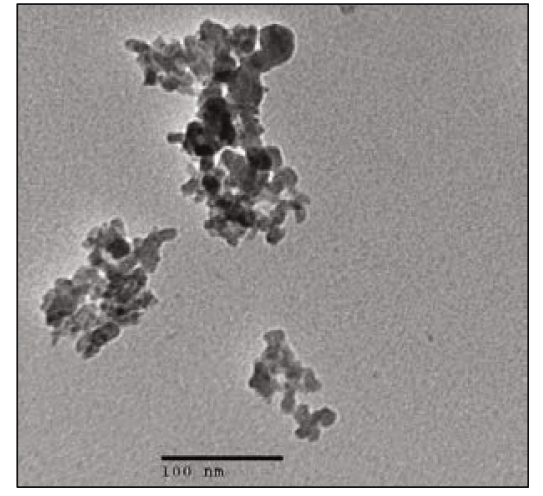

(c)

Figure 10: TEM of CuO NPs (a) first time synthesized, (b) recycled after sonocatalytic degradation, and (c) recycled after photocatalytic degradation.

[34]. The collapse of the bubbles induces high-energy phenomena, i.e., high temperature and pressure $(\sim 5000 \mathrm{~K}$ and 500 bars), electrical discharges, and plasma effects. The consequences of these extreme conditions are the direct thermal dissociation (sonolysis) of dissolved dioxygen and water molecules into highly reactive radical species such as ${ }^{\circ} \mathrm{OH}$, hydrogen $(\mathrm{H})$, and oxygen $(\mathrm{O})$.

$$
\begin{gathered}
\mathrm{H}_{2} \mathrm{O} \stackrel{\text { US|Sonolysis }}{\longrightarrow} \mathrm{OH}^{-}+\mathrm{H}^{\cdot} \\
\mathrm{O}_{2} \stackrel{\text { Us|Sonolysis }}{\longrightarrow} 2 \mathrm{O}^{\circ}
\end{gathered}
$$

These oxygen radicals react with water to form hydroxyl radicals $(\mathrm{OH})$

$$
\mathrm{H}_{2} \mathrm{O}+\mathrm{O} \longrightarrow 2 \mathrm{OH}^{\circ}
$$

Moreover, it has been reported that during the transient collapse of cavitation bubbles, emission of light called "sonoluminescence" also takes place. The spectrum of this emission ranges from the ultraviolet region to well beyond the red end of the visible wavelength range, with a peak at $310 \mathrm{~nm}$. This sonoluminescent spectrum of water is associated with the formation of high-energy species (e.g., excited hydroxyl radicals) from molecular fragmentation of compressed gases, rather than with black body radiation $[35,36]$.

Hence, sonochemistry also involves the emission of light energy for a short period of time, although molecular excitation is thermal, unlike the electronic excitation felt by molecules in photochemical processes. The radiation may lead to the photoexcitation of electrons from the valence band to the conduction band, thus leading to the formation of electron-hole pairs in a similar manner as described above for the photocatalysis (Scheme 2). 


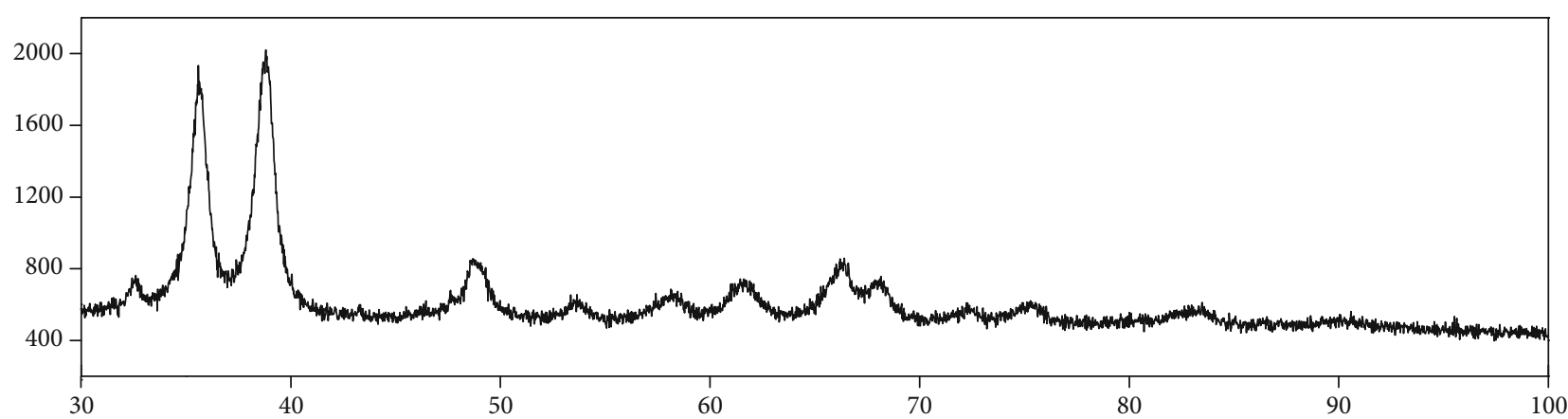

(a)

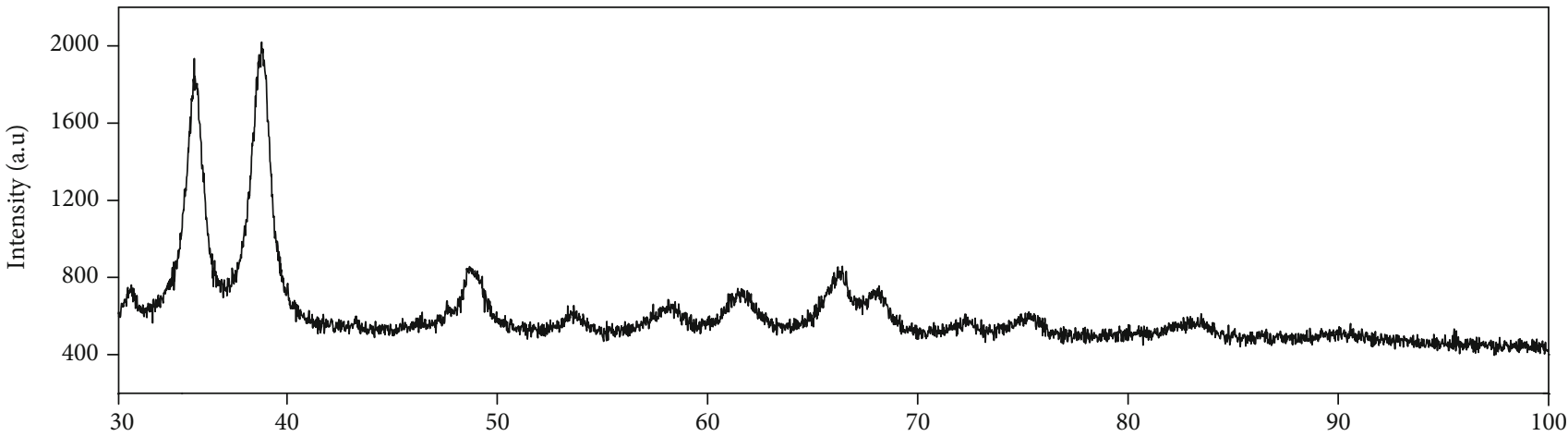

(b)

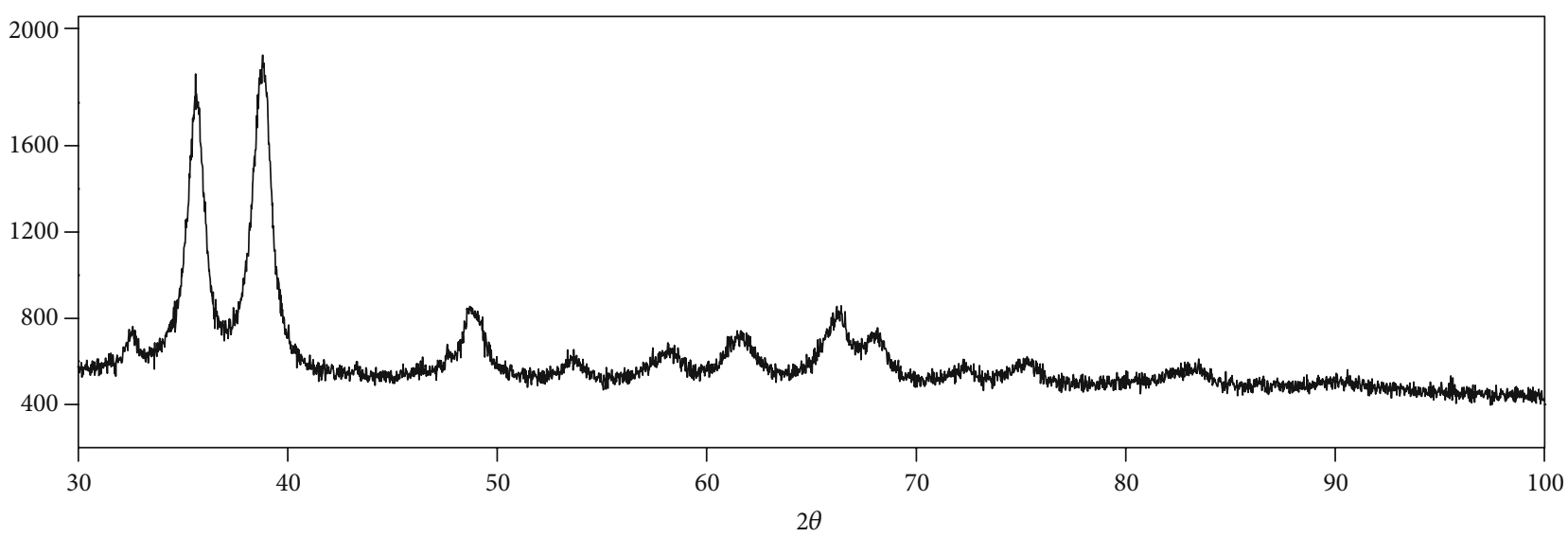

(c)

Figure 11: XRD of CuO NPs (a) first time synthesized, (b) recycled after sonocatalytic degradation, and (c) recycled after photocatalytic degradation.

3.7. Effect of Hydroxyl Radical Scavengers on Degradation of Dyes. To gain insight into the degradation mechanism, experiments were conducted using $\mathrm{OH}$ radical scavengers. As depicted in earlier research, $\mathrm{OH}$ free radical oxidation is a key mechanism in all AOPs $[32,37]$. To measure the quantitative effect of $\mathrm{OH}$ radicals on $\mathrm{VB}$ and $\mathrm{DR}$ degradation, studies were carried out in the presence of DMSO and carbonate ions, which have a hydroxyl radical-scavenging property $[13,38]$. To analyse the effect of scavengers on sonocatalytic degradation and UV-visible photocatalytic degradation of both dyes, experiments were done using $20 \mathrm{ml}$ of $10 \times 10^{-5} \mathrm{M}$ dye concentration and $\mathrm{CuO}$ NP dosage of $25 \mathrm{mg}$ in the presence of radical scavengers. The solution was irradiated with US $(50 \mathrm{~W})$ for 10 minutes and UVvisible irradiation for 20 minutes. It was observed that the degradation efficiency of both the dyes decreased in the presence of radical scavengers (Figure 8). The plausible mechanism in the presence of DMSO and carbonate ion is shown below

$$
\begin{gathered}
\left(\mathrm{CH}_{3}\right)_{2} \mathrm{SO}+\mathrm{OH}^{-} \longrightarrow \mathrm{CH}_{3} \mathrm{SO}_{2} \mathrm{H}+\mathrm{CH}_{3}^{-} \\
\mathrm{CO}_{3}^{2-}+\mathrm{OH}^{-} \longrightarrow \mathrm{CO}_{3}^{-}+\mathrm{OH}^{-}
\end{gathered}
$$

The major decline of degradation efficiency demonstrated that $\mathrm{OH}$ radical plays a vital role in both the 


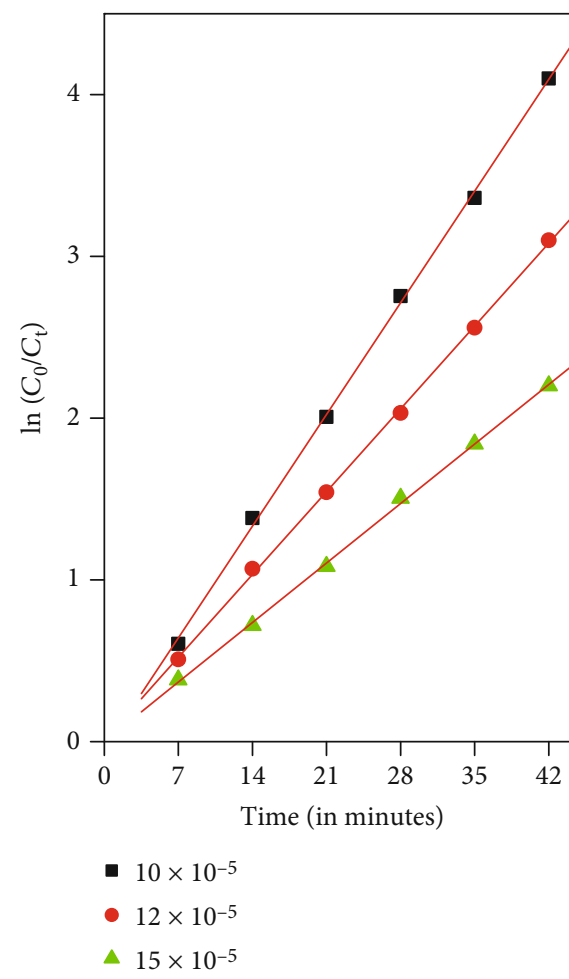

(a)

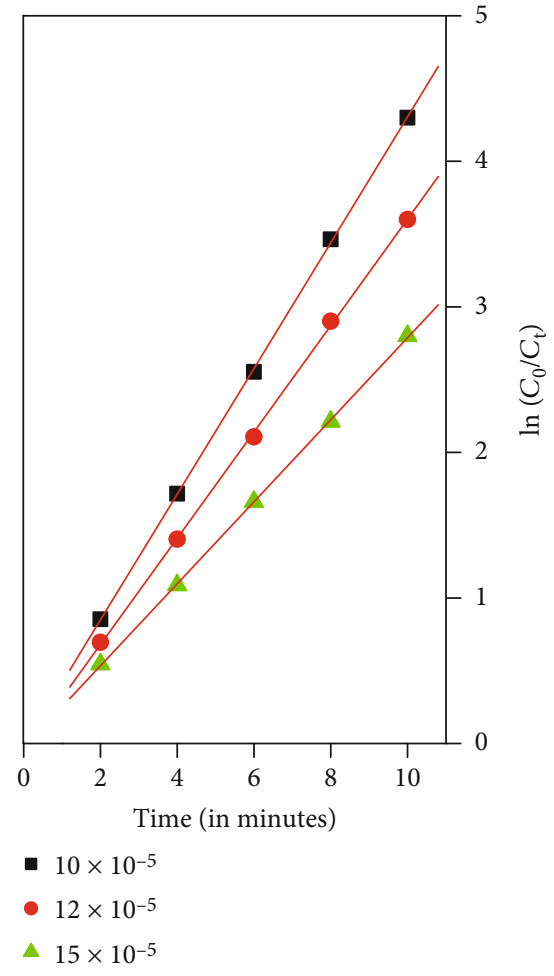

(b)

FIGURE 12: Pseudo-first-order kinetics plot of VB at different concentrations under (a) UV-visible irradiation and (b) US irradiation.

degradation processes and confirms that free radical attack is the dominant controlling mechanism in the degradation of both the DR and VB dyes.

3.8. Reusability of Catalyst. An examination of the sonocatalytic and photocatalytic activities of the recycled $\mathrm{CuO}$ NPs was investigated by collecting the NPs from a sample solution after centrifugation. The separated NPs were thoroughly washed with water and ethanol. The reusability test of $\mathrm{CuO}$ NPs under UV-visible and ultrasonic irradiation on the same organic dyes show the stability of the catalyst. As shown in Figure 9, the reused catalyst showed very little change in the degradation efficiency in both cases, i.e., sonocatalysis and photocatalysis. This emphasizes the excellent chemical stability of the catalyst, making it beneficial for practical wastewater remediation applications. A slight decrease in the photocatalytic activity in each successive cycle can be attributed to several reasons like a degradation of the photocatalyst ( $\mathrm{CuO} \mathrm{NPs}$ ) itself, unavoidable loss of photocatalyst during the recycling processes, and slight aggregation of NPs after the photocatalytic process. To find out why there is a decrease in the activity of used $\mathrm{CuO}$ NPs after 4 cycles, the recycled $\mathrm{CuO}$ NPs have been characterized by TEM and XRD [Figures 10 and 11]. The characteristic XRD diffraction peaks of $\mathrm{CuO}$ NPs are clearly visible and show no notable differences before and after recycling the NPs, indicating that $\mathrm{CuO}$ NPs are chemically stable. In contrast, TEM images of $\mathrm{CuO}$ NPs before and after photocatalytic reaction (4 cycles) (Figure 11) shows the slight aggregation of $\mathrm{CuO}$ NPs, possi- bly by the interactions between $\mathrm{CuO}$ NPs and intermediates formed during the degradation process, thus decreasing the electrostatic energy barrier between NPs and increasing the aggregation behaviour. As these recycled $\mathrm{CuO}$ NPs form clusters, hence, the surface to volume ratio of NPs decreases and their catalytic activity also decreases.

3.9. Comparison of Efficiency of Photocatalysis and Sonocatalysis of $\mathrm{CuO}$ NPs. Competitive analysis of $\mathrm{CuO}$ NPs as sonocatalysts and photocatalysts as shown in Figure 2 reveals that \% decomposition is far better in the case of sonocatalysis. In the presence of $\mathrm{CuO} \mathrm{NPs} \mathrm{and} \mathrm{under} \mathrm{dif-}$ ferent sources of energy, both dyes demonstrated enhanced degradation than without NPs. This is due to an increase in the generation of hydroxyl radicals by enhancing the electron-hole pair formation rate due to the excitation of the catalyst surface by energy source $[5,13]$. To further quantify, kinetics of sonocatalytic and photocatalytic degradation of the $\mathrm{VB}$ and $\mathrm{DR}$ dyes was studied by conducting reactions at an initial concentration $\left(10 \times 10^{-5}, 12 \times 10^{-5}\right.$, and $\left.15 \times 10^{-5}\right)$ with a catalyst dose of $25 \mathrm{mg}$. The graph is plotted for $\ln \left(C_{0} / C_{t}\right)$ vs. the time for photocatalytic and sonocatalytic degradation which resulted in approximately straight lines, as shown in Figures 12 and 13. The kinetic studies were performed on the basis of disappearance of the dye.

The photocatalyst and sonocatalyst with as-prepared samples could be expressed by Langmuir-Hinshelwood mode, Figures 12 and 13. These figures imply that the degradation of $\mathrm{VB}$ and DR in both cases follows a pseudo-first-order kinetics. 


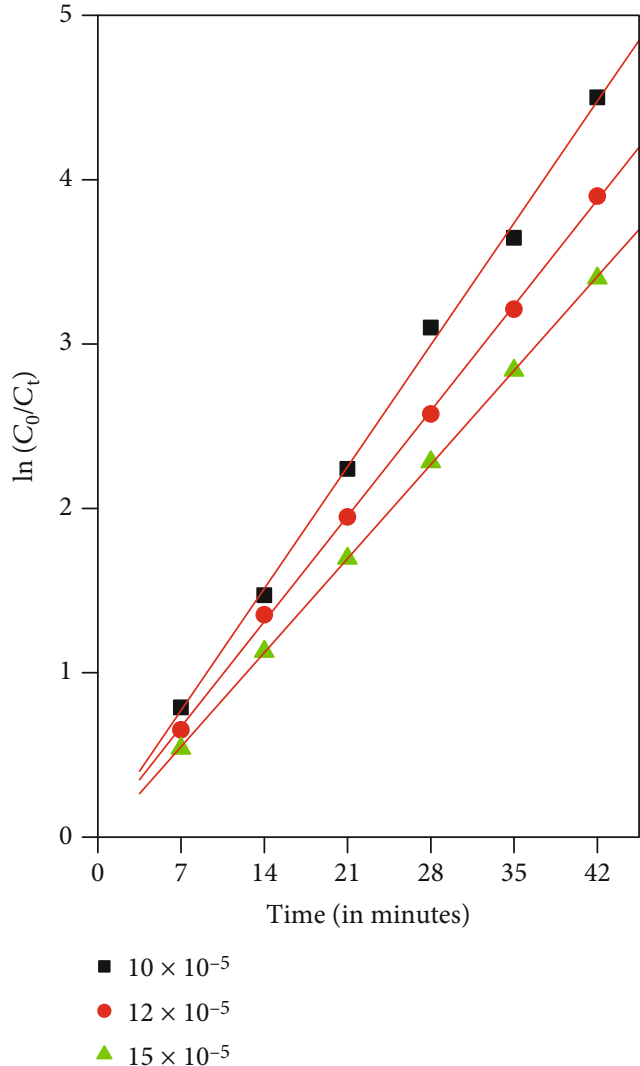

(a)

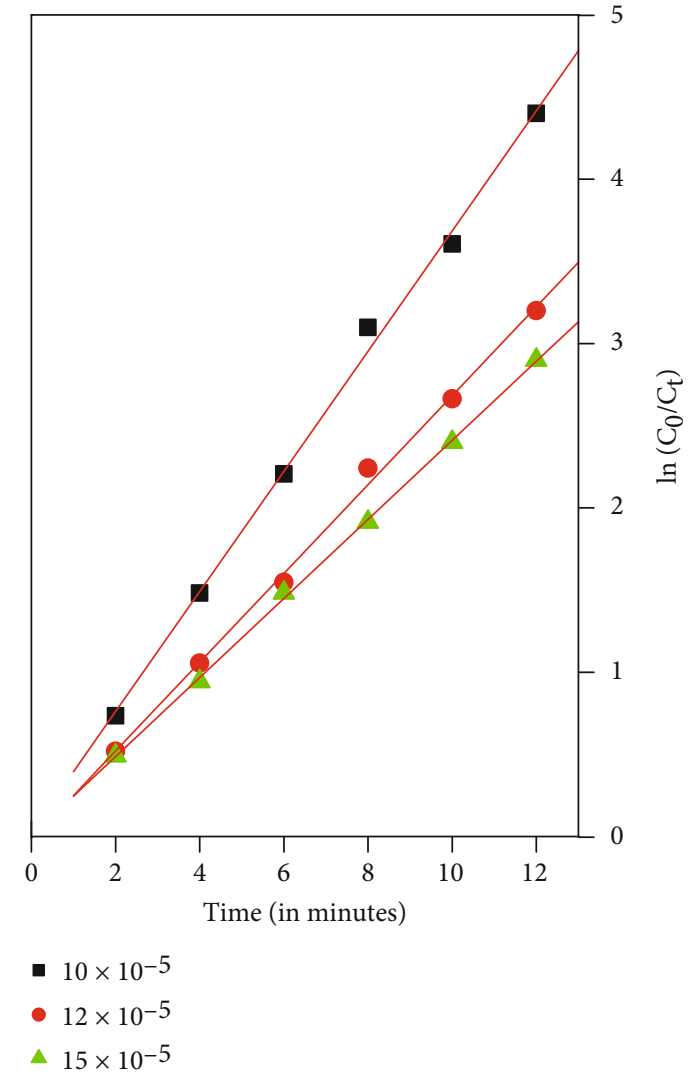

(b)

FIgURE 13: Pseudo-first-order kinetics plot of DR at different concentrations under (a) UV-visible irradiation and (b) US irradiation.

TABLE 2: Pseudo-first-order kinetics rate constants for the degradation of VB and DR under both sources of energy and for different concentration.

\begin{tabular}{|c|c|c|c|c|c|}
\hline Dyes & Dye concentration & Sonocatalysis $k\left(\min ^{-1}\right)$ & Photocatalysis $k\left(\mathrm{~min}^{-1}\right)$ & $R$ sonocatalysis & $R$ photocatalysis \\
\hline \multirow{3}{*}{ Victoria Blue (VB) } & $10 \times 10^{-5} \mathrm{M}$ & 0.43209 & 0.09861 & 0.99992 & 0.99952 \\
\hline & $12 \times 10^{-5} \mathrm{M}$ & 0.36536 & 0.07314 & 0.99975 & 0.99965 \\
\hline & $15 \times 10^{-5} \mathrm{M}$ & 0.28177 & .05255 & 0.99988 & 0.99933 \\
\hline \multirow{3}{*}{ Direct Red (DR) } & $10 \times 10^{-5} \mathrm{M}$ & 0.3656 & 0.10587 & 0.9985 & 0.99888 \\
\hline & $12 \times 10^{-5} \mathrm{M}$ & 0.27036 & 0.09159 & 0.99925 & 0.99962 \\
\hline & $15 \times 10^{-5} \mathrm{M}$ & 0.24064 & 0.08172 & 0.99915 & 0.99811 \\
\hline
\end{tabular}

TABLE 3: Comparison of efficiency of photocatalysis and sonocatalysis of CuO NPs.

\begin{tabular}{llcc}
\hline Dyes & NPs & ${\text { Photocatalysis } k\left(\mathrm{~min}^{-1}\right)}$ & ${\text { Sonocatalysis } k\left(\mathrm{~min}^{-1}\right)}^{-1}$ \\
\hline Victoria Blue $(\mathrm{VB}) 10 \times 10^{-5} \mathrm{M}$ & $\mathrm{CuO}$ & 0.09861 & 0.43209 \\
Direct Red (DR) $10 \times 10^{-5} \mathrm{M}$ & $\mathrm{CuO}$ & 0.10587 & 0.3656 \\
\hline
\end{tabular}

The rate constants are determined by fitting the experimental data in equation (2). The pseudo-first-order reaction rate constant $k$ and regression $R$ calculated from Figures 12 and 13 are given in Table 2 . On comparing the kinetic constant $(k)$ of sonocatalysis with $k$ of photocatalysis for both dyes, it was found that the obtained value of $k$ for the sonocatalyst is much higher than for the photocatalyst. Hence, proving that US irradiations are a better source than UV-visible irradiation for the degradation of dyes in the presence of CuO NPs. CuO NPs have a lower band gap ( $2.3 \mathrm{eV}$ (Figure S5 supplementary data)) so electron-hole recombination is faster and easier. Therefore, the formation of hydroxyl radicals is difficult, which results in lower degradation of dyes in the case of photocatalysis. In the presence of $\mathrm{CuO}$ NPs, the sonocatalytic efficiency to decompose organic pollutants was enhanced. This is due to synergistic effects of ultrasound and solid catalyst, namely [39], (i) added powders could provide additional nuclei for cavitation bubble formation, (ii) US irradiation enhances the 
mass transfer of organic pollutants between the liquid phase and the catalyst surface, (iii) US irradiation increases the active surface area due to ultrasound deaggregating, and (iv) the catalyst can be excited by ultrasound-induced luminescence which has a wide wavelength and increased production of $\mathrm{OH}$ in the reaction mixture (Ghosh et al., 2013). Thus, sonocatalysis seems to enhance the degradation ratio of organic pollutants due to the increase in the generation of $\mathrm{OH}$.

3.10. Comparison of Efficiency of Photocatalysis and Sonocatalysis of $\mathrm{CuO}$ NPs. On comparing the photocatalytic and sonocatalytic activities $\mathrm{CuO}$ NPs, it was found that the $\mathrm{CuO}$ NPs are superior as sonocatalysts than the photocatalysts (Table 3 ). This may be attributed to the presence of a smaller band gap of $\mathrm{CuO} \mathrm{NPs}(2.3 \mathrm{eV}$ in the present case) which enables the electron-hole recombination to be less or slower, so there is more formation of ROS for the degradation of dyes. Additionally, the CuO NPs being better as sonocatalysts is because of the higher surface area calculated by BET of CuO NPs $\left(49.33 \mathrm{~m}^{2} \mathrm{~g}^{-1}\right)$. Higher surface area leads to the formation of more cavitation bubbles, which, in turn, produces more local temperature and pressure effects and eventually creates more ROS for the degradation of dyes.

\section{Conclusion}

In summary, we have demonstrated the comparison of the catalytic effect of $\mathrm{CuO}$ NPs on the degradation of the cationic (VB) and anionic (DR) dyes under different energy sources, i.e., UV-visible irradiation and US radiations. The synergistic effect of $\mathrm{CuO}$ NPs and sonocatalysis has been demonstrated to be more effective in degrading both dyes as compared to $\mathrm{CuO}$ NPs and photocatalysis. High catalyst dosage and low initial dye concentration were favoured for the sonocatalytic or photocatalytic degradation of both dyes. Degradation follows the formation of a free radical mechanism, which is further verified by the addition of radical scavengers which reduced the sonocatalytic or photocatalytic degradation. Comparison of the efficiency of photocatalysis and sonocatalysis of $\mathrm{CuO}$ NPs was examined and reported. Overall, the application of $\mathrm{CuO}$ NPs can be a promising and efficient approach for the sonocatalysis of both the cationic and anionic dyes with high reusability potential.

\section{Data Availability}

Data of all by measurements can be available with the authors and shared upon request. Data are stored in secured devices at the author's institutions. Data can be used to reproduce the results and also generate new sets of results. The authors have full access to these data and full permissions to make it available public for greater use to the scientific community.

\section{Conflicts of Interest}

The authors declare that they have no conflicts of interest.

\section{Acknowledgments}

Authors would like to acknowledge the support of UGC, India under INDO-US $21^{\text {st }}$ Century Knowledge Initiative Project (F. No. 194-2/2016 (IC)). SS would like to acknowledge the USA funding sources, Florida Industrial and Phosphate Research Institute Grant (GR-1800010) and Hinkley Center for Solid and Hazardous Waste Management. The authors also acknowledge the support of SAIF/CIL, Chandigarh, for providing instrumentation facilities.

\section{Supplementary Materials}

Figure S1 represents the X-ray diffraction pattern of the assynthesized nanoparticles, where it is composed of a pure $\mathrm{CuO}$ phase with a monoclinic structure without any peaks of impurity. The particle size, as calculated from FWHM of reflection (111/200) of a monoclinic $\mathrm{CuO}$ structure using the Debye Scherrer formula was found to be $18 \mathrm{~nm}$. For determining the morphology, TEM micrograph of $\mathrm{CuO}$ NPs was taken and the NPs were observed to be almost spherical in shape, well dispersed, and within a narrow range of size distribution (17-22 $\mathrm{nm}$ ) as demonstrated in Figures S2 and S3 below [28]. The zeta potential of the CuO NP dispersions was measured over $\mathrm{pH}$ values ranging from 2 to 10 , and the corresponding pHzpc was found to be $\sim 8.5$ as shown in Figure S4. The lower band gap of $\mathrm{CuO}$ NPs $(\sim 2.3 \mathrm{eV})$ as obtained from the Tauc plot is depicted in Figure S5, and it leads for faster and easier electron-hole recombination processes during the sonocatalytic and photocatalytic processes. Supplementary Table S1-S4. Tables S1-S4 are the statistical analysis data ( $P$ value) obtained via the ANOVA for the effect of catalyst dose on the degradation of DR and VB dyes under UV-Vis and US irradiations. (Supplementary Materials)

\section{References}

[1] P. Srivastava, S. Goyal, and P. K. Patnala, "Degradation of reactive, acid and basic textile dyes in the presence of ultrasound and rare earths [lanthanum and praseodymium]," Ultrasonics Sonochemistry, vol. 21, no. 6, pp. 1994-2009, 2014.

[2] S. Chaudhary, Y. Kaur, B. Jayee, G. R. Chaudhary, and A. Umar, "NiO nanodisks: highly efficient visible-light driven photocatalyst, potential scaffold for seed germination of Vigna radiata and antibacterial properties," Journal of Cleaner Production, vol. 190, pp. 563-576, 2018.

[3] S. A. Larik, A. Khatri, S. Ali, and S. H. Kim, "Batchwise dyeing of bamboo cellulose fabric with reactive dye using ultrasonic energy," Ultrasonics Sonochemistry, vol. 24, no. 2015, pp. 178-183, 2015.

[4] J. R. Steter, W. R. P. Barros, M. R. V. Lanza, and A. J. Motheo, "Electrochemical and sonoelectrochemical processes applied to amaranth dye degradation," Chemosphere, vol. 117, pp. 200-207, 2014.

[5] S. S. Srinivasan, J. Wade, and E. K. Stefanakos, "Visible light photocatalysis via CdS/TiO2 nanocomposite materials," Journal of Nanomaterials, vol. 2006, article 87326, 7 pages, 2006.

[6] A. Karci, "Degradation of chlorophenols and alkylphenol ethoxylates, two representative textile chemicals, in water by advanced oxidation processes: the state of the art on 
transformation products and toxicity," Chemosphere, vol. 99, pp. 1-18, 2014.

[7] S. Chaudhary, Y. Kaur, A. Umar, and G. R. Chaudhary, "1butyl-3-methylimidazolium tetrafluoroborate functionalized $\mathrm{ZnO}$ nanoparticles for removal of toxic organic dyes," Journal of Molecular Liquids, vol. 220, pp. 1013-1021, 2016.

[8] K. Govindan, M. Raja, S. U. Maheshwari, and M. Noel, “Analysis and understanding of amido black 10B dye degradation in aqueous solution by electrocoagulation with the conventional oxidants peroxomonosulfate, peroxodisulfate and hydrogen peroxide," Environmental Science: Water Research \& Technology, vol. 1, no. 1, pp. 108-119, 2015.

[9] P. Sathishkumar, R. V. Mangalaraja, O. Rozas et al., "Sonophotocatalytic degradation of Acid Blue 113 in the presence of rare earth nanoclusters loaded $\mathrm{TiO}_{2}$ nanophotocatalysts," Separation and Purification Technology, vol. 133, pp. 407-414, 2014.

[10] S. S. Srinivasan, J. Wade, and E. K. Stefanakos, "Synthesis and characterization of photocatalytic $\mathrm{TiO} 2-\mathrm{ZnFe} 2 \mathrm{O} 4$ nanoparticles," Journal of Nanomaterials, vol. 2006, article 45712, 4 pages, 2006.

[11] P. Saharan, G. R. Chaudhary, S. Lata, S. K. Mehta, and S. Mor, "Ultra fast and effective treatment of dyes from water with the synergistic effect of $\mathrm{Ni}$ doped $\mathrm{ZnO}$ nanoparticles and ultrasonication," Ultrasonics Sonochemistry, vol. 22, pp. 317-325, 2015.

[12] D. Rajamanickam, P. Dhatshanamurthi, and M. Shanthi, "Preparation and characterization of $\mathrm{SeO}_{2} / \mathrm{TiO}_{2}$ composite photocatalyst with excellent performance for sunset yellow azo dye degradation under natural sunlight illumination," Spectrochimica Acta Part A: Molecular and Biomolecular Spectroscopy, vol. 138, pp. 489-498, 2015.

[13] A. Khataee, A. Karimi, S. Arefi-Oskoui et al., "Sonochemical synthesis of Pr-doped $\mathrm{ZnO}$ nanoparticles for sonocatalytic degradation of Acid Red 17," Ultrasonics Sonochemistry, vol. 22, pp. 371-381, 2015.

[14] H. Y. He and J. Lu, "Highly photocatalytic activities of magnetically separable reduced graphene oxide- $\mathrm{CoFe}_{2} \mathrm{O}_{4}$ hybrid nanostructures in dye photodegradation," Separation and Purification Technology, vol. 172, pp. 374-381, 2017.

[15] M. L. A. Kumari and L. G. Devi, "New insights into the origin of the visible light photocatalytic activity of Fe (III) porphyrin surface anchored $\mathrm{TiO}_{2}$," Environmental Science: Water Research and Technology, vol. 1, pp. 177-187, 2015.

[16] S. S. Srinivasan, S. L. Wallen, and J. Douyon, "Synergistic chemical oxidative and photocatalytic enhancer system (scopes) for wastewater remediation," 2018, US Patent $16 / 425,590$.

[17] C. Li, S. Srinivasan, N. Kislov, M. Schmidt, E. K. Stefanakos, and D. Y. Goswami, "Enhancement of $\mathrm{TiO}_{2}$ photocatalytic activity by $\mathrm{N}$ - doping using the gas phase impregnation method," MRS Proceedings, vol. 1217, pp. Y03-Y35, 2009.

[18] R. I. MacCuspie, H. Hyman, C. Yakymyshyn, S. S. Srinivasan, J. Dhau, and C. Drake, "A framework for identifying performance targets for sustainable nanomaterials," Sustainable Materials and Technologies, vol. 1-2, pp. 17-25, 2014.

[19] S. Wang, X. Zhang, L. Pan et al., "Controllable sonochemical synthesis of $\mathrm{Cu}_{2} \mathrm{O} / \mathrm{Cu}_{2}(\mathrm{OH})_{3} \mathrm{NO}_{3}$ composites toward synergy of adsorption and photocatalysis," Applied Catalysis B: Environmental, vol. 164, pp. 234-240, 2015.

[20] A. Bhattacharjee, M. Ahmaruzzaman, and T. Sinha, "Surfactant effects on the synthesis of durable tin-oxide nanoparticles and its exploitation as a recyclable catalyst for the elimination of toxic dye: a green and efficient approach for wastewater treatment," RSC Advances, vol. 4, no. 93, pp. 51418-51429, 2014.

[21] D. Yang, J. Zhao, H. Liu et al., "Enhancing photoactivity of $\mathrm{TiO} 2(\mathrm{~B}) /$ anatase core-shell nanofibers by selectively doping cerium ions into the TiO2(B) core," Chemistry - A European Journal, vol. 19, no. 16, pp. 5113-5119, 2013.

[22] R. Abazari, A. R. Mahjoub, and S. Sanati, "A facile and efficient preparation of anatase titania nanoparticles in micelle nanoreactors: morphology, structure, and their high photocatalytic activity under UV light illumination," RSC Advances, vol. 4, no. 99, pp. 56406-56414, 2014.

[23] F. Mirhoseini and A. Salabat, "Ionic liquid based microemulsion method for the fabrication of poly(methyl methacrylate)-TiO2nanocomposite as a highly efficient visible light photocatalyst," RSC Advances, vol. 5, no. 17, pp. 1253612545, 2015.

[24] A. R. Khataee, M. Zarei, and R. Ordikhani-Seyedlar, "Heterogeneous photocatalysis of a dye solution using supported $\mathrm{TiO}_{2}$ nanoparticles combined with homogeneous photoelectrochemical process: molecular degradation products," Journal of Molecular Catalysis A: Chemical, vol. 338, pp. 84-91, 2011.

[25] A. Khataee, R. Darvishi, R. D. C. Soltani, A. Karimi, and S. W. Joo, "Sonocatalytic degradation of a textile dye over Gd-doped $\mathrm{ZnO}$ nanoparticles synthesized through sonochemical process," Ultrasonics Sonochemistry, vol. 23, pp. 219-230, 2011.

[26] N. A. Fernandez, L. Rodriguez-Freire, M. Keswani, and R. Sierra-Alvarez, "Effect of chemical structure on the sonochemical degradation of perfluoroalkyl and polyfluoroalkyl substances (PFASs)," Environmental Science: Water Research \& Technology, vol. 2, no. 6, pp. 975-983, 2016.

[27] N. Kaur, G. Kaur, A. Bhalla, J. S. Dhau, and G. R. Chaudhary, "Metallosurfactant based Pd-Ni alloy nanoparticles as a proficient catalyst in the Mizoroki Heck coupling reaction," Green Chemistry, vol. 20, no. 7, pp. 1506-1514, 2018.

[28] G. R. Chaudhary, P. Bansal, N. Kaur, and S. K. Mehta, "Recyclable $\mathrm{CuO}$ nanoparticles as heterogeneous catalysts for the synthesis of xanthenes under solvent free conditions," RSC Advances, vol. 4, no. 90, pp. 49462-49470, 2014.

[29] B. P. Nenavathu, A. V. R. Krishna Rao, A. Goyal, A. Kapoor, and R. K. Dutta, "Synthesis, characterization and enhanced photocatalytic degradation efficiency of Se doped $\mathrm{ZnO}$ nanoparticles using trypan blue as a model dye," Applied Catalysis A: General, vol. 459, pp. 106-113, 2013.

[30] H. T. Chandran, S. Thangavel, C. V. Jipsa, and G. Venugopal, "Study on inorganic oxidants assisted sonocatalytic degradation of Resazurin dye in presence of $\beta-\mathrm{SnWO}_{4}$ nanoparticles," Materials Science in Semiconductor Processing, vol. 27, pp. 212-219, 2014.

[31] A. Raizada, D. Ganguly, and M. M. Mankad, "A highly efficient copper oxide nanopowder for adsorption of methylene blue dye from aqueous medium," Journal of Chemical Engineering Research, vol. 2, pp. 249-258, 2014.

[32] A. Nezamzadeh-Ejhieh and Z. Salimi, "Solar photocatalytic degradation of o-phenylenediamine by heterogeneous $\mathrm{CuO} / \mathrm{X}$ zeolite catalyst," Desalination, vol. 280, no. 1-3, pp. 281-287, 2011.

[33] N. Talebian, M. R. Nilforoushan, and F. J. Mogaddas, "Comparative study on the sonophotocatalytic degradation of hazardous waste," Ceramics International, vol. 39, no. 5, pp. 4913-4921, 2013. 
[34] T. Ghosh, K. Ullah, V. Nikam, C. Y. Park, Z. D. Meng, and W. C. Oh, "The characteristic study and sonocatalytic performance of CdSe-graphene as catalyst in the degradation of azo dyes in aqueous solution under dark conditions," Ultrasonics Sonochemistry, vol. 20, no. 2, pp. 768-776, 2013.

[35] T. Philippe, P. Pichat, C. Guillard, P. Christian, and T. Chopin, "Degradation of phenyltrifluoromethylketone in water by separate or simultaneous use of $\mathrm{TiO}_{2}$ photocatalysis and 30 or 515 $\mathrm{kHz}$ ultrasound," Physical Chemistry Chemical Physics, vol. 1, no. 19, pp. 4663-4668, 1999.

[36] C. Balaji, V. S. Moholkar, A. B. Pandit, and M. Ashokkumar, "Mechanistic investigations on sonophotocatalytic degradation of textile dyes with surface active solutes," Industrial and Engineering Chemistry Research, vol. 50, no. 20, pp. 1148511494, 2011.

[37] A. R. Khataee and M. Zarei, "Photocatalysis of a dye solution using immobilized $\mathrm{ZnO}$ nanoparticles combined with photoelectrochemical process," Desalination, vol. 273, no. 2-3, pp. 453-460, 2011.

[38] M. J. Burkitt and R. P. Mason, "Direct evidence for in vivo hydroxyl-radical generation in experimental iron overload: an ESR spin-trapping investigation," Proceedings of the National Academy of Sciences, vol. 88, no. 19, pp. 8440-8444, 1991.

[39] S. E. Karekar, B. A. Bhanvase, S. H. Sonawane, M. P. Deosarkar, D. V. Pinjari, and A. B. Pandit, "Synthesis of zinc molybdate and zinc phosphomolybdate nanopigments by an ultrasound assisted route: advantage over conventional method," Chemical Engineering and Processing, vol. 87, pp. 51-59, 2015. 